\title{
COMPUTATION OF 3D VERTEX SINGULARITIES FOR LINEAR ELASTICITY: ERROR ESTIMATES FOR A FINITE ELEMENT METHOD ON GRADED MESHES *
}

\begin{abstract}
Thomas Apel ${ }^{1}$, Anna-Margarete Sändig ${ }^{2}$ And Sergey I. Solov’ev ${ }^{3}$
Abstract. This paper is concerned with the computation of 3D vertex singularities of anisotropic elastic fields with Dirichlet boundary conditions, focusing on the derivation of error estimates for a finite element method on graded meshes. The singularities are described by eigenpairs of a corresponding operator pencil on spherical polygonal domains. The main idea is to introduce a modified quadratic variational boundary eigenvalue problem which consists of two self-adjoint, positive definite sesquilinear forms and a skew-Hermitean form. This eigenvalue problem is discretized by a finite element method on graded meshes. Based on regularity results for the eigensolutions estimates for the finite element error are derived both for the eigenvalues and the eigensolutions. Finally, some numerical results are presented.
\end{abstract}

Mathematics Subject Classification. 65N25, 65N30, 74G70.

Received: December 21, 2001. Revised: June 3, 2002.

\section{INTRODUCTION}

The present paper is concerned with the study of the behaviour of three-dimensional elastic fields satisfying Dirichlet boundary conditions near the vertex of a polyhedral cone. It is well known, that stress singularities can arise in the neighborhood of the vertex $[9,34,40]$. The detailed knowledge of the singular terms of the elastic fields is of interest, e.g., in crack mechanics where the intersection of crack fronts or notches with the surface of the body generates vertices. Moreover, in computational mechanics, the lack of regularity near edges or corners demands modified discretization procedures. Our goals are to describe a mathematical method which leads to an efficient computation of the vertex singularities in very general situations, to derive error estimates for finite element solutions and to present numerical results.

Keywords and phrases. Quadratic eigenvalue problems, linear elasticity, 3D vertex singularities, finite element methods, error estimates.

* The authors were supported by Deutsche Forschungsgemeinschaft (German Research Foundation), Sonderforschungsbereiche (Collaborative Research Centers) 393 and 404.

1 TU Chemnitz, Fakultät für Mathematik, 09107 Chemnitz, Germany. e-mail: apel@mathematik.tu-chemnitz.de

${ }^{2}$ Universität Stuttgart, Mathematisches Institut A, Pfaffenwaldring 57, 70511 Stuttgart, Germany.

e-mail: saendig@mathematik.uni-stuttgart.de

${ }^{3}$ Kazan State University, Faculty of computer science and cybernetics, Kremlevskaya 18, 420008 Kazan, Russia.

e-mail: sergei.solovyev@ksu.ru 
Writing the boundary value problems in spherical coordinates $(r, \varphi, \theta)$ centered in the vertex of the cone and using the Mellin-transformation $\left(r \partial_{r} \rightarrow \alpha\right)$ we get a parameter-depending boundary value problem $\mathcal{A}_{c}(\alpha) u=\mathcal{F}$ in a curved polygon $\tilde{\Omega} \subset S^{2}$ on the unit sphere $S^{2} \subset \mathbb{R}^{3}$. The eigenvalues $\alpha_{i}$ and the eigensolutions $u\left(\alpha_{i} ; \cdot\right)$ of the operator pencil $\mathcal{A}_{c}(\cdot)$ generate the singular terms of the elastic fields near the vertex. They have the form (here written without logarithmic terms)

$$
\sum_{i} c_{i} r^{\alpha_{i}} u\left(\alpha_{i} ; \varphi, \theta\right)
$$

In order to solve this generalized eigenvalue problem efficiently we formulate a modified quadratic eigenvalue problem by introducing the parameter $\lambda=\alpha+1 / 2$ leading to an eigenvalue problem with a symmetric distribution of the eigenvalues. It reads in the weak formulation: Find $\lambda \in \mathbb{C}$ and $u \in V \backslash\{0\}$ such that

$$
k(u, v)=\lambda g(u, v)+\lambda^{2} m(u, v) \quad \forall v \in V
$$

with self-adjoint, positive definite forms $k(\cdot, \cdot)$ and $m(\cdot, \cdot)$ and a skew-Hermitean form $g(\cdot, \cdot)$. By $V$ we denote a complex Hilbert space of vector functions defined on $\tilde{\Omega}$. For the numerical solution we construct a finite element subspace $V_{h} \subset V$ and look for the finite element solution of problem (1): Find $\lambda_{h} \in \mathbb{C}$ and $u_{h} \in V_{h} \backslash\{0\}$ such that

$$
k\left(u_{h}, v_{h}\right)=\lambda_{h} g\left(u_{h}, v_{h}\right)+\lambda_{h}^{2} m\left(u_{h}, v_{h}\right) \quad \forall v_{h} \in V_{h} .
$$

This approach is widely used in the engineering literature [28,47]. Modern methods to solve the algebraic eigenvalue problem (2) by exploiting the structure can be found, for example, in $[3,7,38]$.

The main interest of the current paper is to investigate the finite element errors $\left|\lambda-\lambda_{h}\right|$ and $\left\|u-u_{h}\right\|_{V}$. The particular difficulty is that the domain $\tilde{\Omega}$ has, in general, corners such that the eigenfunctions are not smooth. In Section 2, we formulate the quadratic boundary-eigenvalue problems at the sphere. They are related to the three-dimensional boundary value problems for the elasticity operator. Furthermore, we define Hilbert spaces on the spherical domain $\tilde{\Omega}$, sesquilinear forms and formulate the modified variational quadratic eigenvalue problem with the parameter $\lambda$. We also describe basic properties of the sesquilinear forms. In Section 3, we introduce more notation and derive regularity results for eigensolutions of two related operator pencils. These results are in principle well known but here we need modified statements which are necessary for the proof of the approximation results.

Section 4 is devoted to error estimates for the approximate eigenvalue problem. We start with some general approximation results in Section 4.1. Then we introduce graded meshes which are appropriate for the approximation of the eigenfunctions with piecewise linear finite elements. In order to get approximation error estimates some non-standard local interpolation error estimates are proved in Section 4.3. The main challenge lay in the use of appropriate weighted Sobolev spaces due to the transformation of the eigenvalue problem from the sphere into the plane parameter domain and due to the reduced regularity of the eigenfunctions. Together with the abstract results of Section 4.1 we conclude approximation error estimates for the eigenvalues and eigenfunctions. The estimates are optimal only due to the use of the graded meshes. The main result is that an appropriately calculated approximate eigenvalue $\hat{\lambda}_{h}$ is second order accurate, $\left|\lambda-\hat{\lambda}_{h}\right| \lesssim h^{2}$, where $h$ is the global mesh size which relates to the number $N$ of degrees of freedom by $N \sim h^{-2}$. Numerical results are discussed in Section 5 . They confirm the theoretically predicted convergence orders.

For simplicity we consider Dirichlet boundary conditions; other boundary conditions as Neumann or mixed boundary conditions can be treated similarly [24].

We use the notation $a \lesssim b$ and $a \sim b$ which means the existence of positive constants $C_{1}$ and $C_{2}$ (which are independent of the discretization parameter $h$ and of the function under consideration) such that $a \leq C_{2} b$ and $C_{1} b \leq a \leq C_{2} b$, respectively. 


\section{Formulation of the eigenvalue problem AND REVIEW of KNOWN Results}

\subsection{Description of corner singularities by an operator eigenvalue problem}

The equilibrium equations for linear anisotropic fields in a polyhedral domain $G$ read

$$
-\sum_{j=1}^{3} \partial_{j} \sigma_{i j}=F_{i} \quad \text { in } G, \quad i=1,2,3
$$

where $F_{i}$ are given body forces, $\boldsymbol{\sigma}=\left(\sigma_{i j}\right)_{i, j=1}^{3}$ is the stress tensor, $x=\left(x_{1}, x_{2}, x_{3}\right)$ are Cartesian coordinates, and $\partial_{j}:=\partial / \partial x_{j}$. Denote by $U=\left(U_{1}, U_{2}, U_{3}\right)^{\top}$ the displacement field and by $\varepsilon(U)=\left(\varepsilon_{l n}(U)\right)_{l, n=1}^{3}$ the associated linearized strain tensor where $\varepsilon_{l n}(U)=\frac{1}{2}\left(\partial_{l} U_{n}+\partial_{n} U_{l}\right)$. For small strains, Hooke's law yields

$$
\sigma_{i j}(U)=\sum_{l, n=1}^{3} a_{i j l n} \varepsilon_{l n}(U), \quad i, j, l, n=1,2,3
$$

The elastic moduli $a_{i j m n}$ are real valued constants and satisfy the symmetry relations

$$
a_{i j l n}=a_{l n i j}=a_{j i l n}=a_{i j n l}
$$

The energy conservation law yields a strong ellipticity and boundedness condition for the corresponding quadratic form

$$
M_{1} \sum_{i, j=1}^{3}\left|\xi_{i j}\right|^{2} \leq \sum_{i, j, l, n=1}^{3} a_{i j l n} \xi_{i j} \xi_{l n} \leq M_{2} \sum_{i, j=1}^{3}\left|\xi_{i j}\right|^{2} \quad \forall \xi_{i j} \in \mathbb{R}, i, j=1,2,3 .
$$

It follows that the elastic matrix $\mathbf{A}$ is symmetric and positive definite,

$$
\mathbf{A}=\left(\begin{array}{cccccc}
a_{1111} & a_{1122} & a_{1133} & a_{1123} & a_{1131} & a_{1112} \\
\cdot & a_{222} & a_{2233} & a_{2223} & a_{2231} & a_{2212} \\
\cdot & \cdot & a_{3333} & a_{3323} & a_{3331} & a_{3312} \\
\cdot & \cdot & \cdot & a_{2323} & a_{2331} & a_{2312} \\
\cdot & \cdot & \cdot & \cdot & a_{3131} & a_{3112} \\
\cdot & \cdot & \cdot & \cdot & \cdot & a_{1212}
\end{array}\right)
$$

with

$$
D^{\top}=\left(\begin{array}{cccccc}
\partial_{1} & 0 & 0 & 0 & \partial_{3} & \partial_{2} \\
0 & \partial_{2} & 0 & \partial_{3} & 0 & \partial_{1} \\
0 & 0 & \partial_{3} & \partial_{2} & \partial_{1} & 0
\end{array}\right)
$$

we obtain $D U=\left(\varepsilon_{11}, \varepsilon_{22}, \varepsilon_{33}, 2 \varepsilon_{23}, 2 \varepsilon_{31}, 2 \varepsilon_{12}\right)^{\top}$ and $\mathbf{A} D U=\left(\sigma_{11}, \sigma_{22}, \sigma_{33}, \sigma_{23}, \sigma_{31}, \sigma_{12}\right)^{\top}$. With this notation, we get the linear elasticity equations (3) in the form $-L U=F$ with $L=D^{\top} \mathbf{A} D$.

In the following we will study the homogeneous Dirichlet problem

$$
-L U=F \quad \text { in } G, \quad U=0 \quad \text { on } \partial G
$$


The variational formulation of the problem is to find $U \in H_{0}^{1}(G)^{3}$ for given $F \in L^{2}(G)^{3}$ such that

$$
\int_{G}(D V)^{\top} \mathbf{A} D U \mathrm{~d} x=\int_{G} F \cdot V \mathrm{~d} x \quad \forall v \in H_{0}^{1}(G)^{3},
$$

with $\mathbf{A}$ and $D$ from (7) and (8), respectively.

The behaviour of elastic fields near vertices of the polyhedron $G$ can be locally investigated by means of a partition of unity. Therefore we can consider the problem in an infinite cone $K \subset R^{3}$ with vertex $O$ at the origin, i.e.

$$
K=\left\{x \in \mathbb{R}^{3}: 0<|x|<\infty, x /|x| \in \tilde{\Omega}\right\},
$$

where $\tilde{\Omega}$ is a subdomain of the unit sphere $S^{2} \subset \mathbb{R}^{3}$ with Lipschitz boundary $\partial \tilde{\Omega}$. We introduce spherical coordinates $(r, \varphi, \theta)$ centered in $O$. Vertex singularities are searched in the form

$$
r^{\alpha} u(\alpha ; \varphi, \theta)
$$

The functions (11) have to be a solution of the homogeneous system

$$
L U=0 \quad \text { in } K, \quad U=0 \quad \text { on } \partial K .
$$

In other words, $u(\alpha ; \cdot)$ is solution of

$$
\mathcal{A}_{c}(\alpha) u(\alpha ; \cdot)=0 \quad \text { in } \tilde{\Omega},
$$

where $\mathcal{A}_{c}(\alpha)$ is the operator pencil which is obtained by inserting (11) into our homogeneous problem in the cone $K$ and using the Mellin transform

$$
u(\alpha ; \varphi, \theta):=\int_{0}^{\infty} r^{-\alpha} U(r, \varphi, \theta) \frac{\mathrm{d} r}{r}
$$

which maps $r \partial_{r}$ into the complex parameter $\alpha, \partial_{r}:=\partial / \partial r$. We will discuss this in more detail in Section 3.2. The problem (12) is a quadratic eigenvalue problem which has a finite number of eigenvalues in any strip $c_{1} \leq \operatorname{Re} \alpha \leq c_{2}$. The set of these eigenvalues is discrete, see also Section 3.3 below.

\subsection{Function spaces and weak formulation of the problem}

For the weak formulation of the quadratic eigenvalue problem (12) we introduce now appropriate function spaces. We consider $\tilde{\Omega} \subset S^{2}$ in spherical coordinates $(\varphi, \theta)$ and Cartesian coordinates $x=\left(x_{1}, x_{2}, x_{3}\right)=$ $(\cos \varphi \sin \theta, \sin \varphi \sin \theta, \cos \theta)$, and denote by $\Omega \subset \mathbb{R}^{2}$ the corresponding domain in the parameter plane,

$$
\tilde{\Omega}=\left\{(\cos \varphi \sin \theta, \sin \varphi \sin \theta, \cos \theta) \in \mathbb{R}^{3}:(\varphi, \theta) \in \Omega\right\} .
$$

In this sense we write $v(\varphi, \theta)=\tilde{v}(\cos \varphi \sin \theta, \sin \varphi \sin \theta, \cos \theta)$. We also use two different norm symbols to define the complex Lebesgue space $L^{2}$,

$$
\|\tilde{v}\|_{0, \tilde{\Omega}}^{2}:=\int_{\tilde{\Omega}}|\tilde{v}|^{2} \mathrm{~d} S, \quad|[v]|_{0, \Omega}^{2}:=\int_{\Omega}|v|^{2} \mathrm{~d} \omega, \quad \mathrm{d} \omega:=\sin \theta \mathrm{d} \theta \mathrm{d} \varphi .
$$


Moreover, let $H^{1}$ and $H^{2}$ be the complex Sobolev spaces endowed with the norms (expressed in the parameter domain $\Omega$ )

$$
\begin{aligned}
|[v]|_{k, \Omega}^{2} & :=|[v]|_{0, \Omega}^{2}+\sum_{j=1}^{k}[v]_{j, \Omega}^{2}, \quad k=1,2 \\
{[v]_{1, \Omega}^{2} } & :=\int_{\Omega}\left(\left|\sin ^{-1} \theta \partial_{\varphi} v\right|^{2}+\left|\partial_{\theta} v\right|^{2}\right) \mathrm{d} \omega \\
{[v]_{2, \Omega}^{2} } & :=\int_{\Omega}\left(\left|\sin ^{-2} \theta \partial_{\varphi \varphi} v\right|^{2}+\left|\sin ^{-1} \theta \partial_{\varphi \theta} v\right|^{2}+\left|\partial_{\theta \theta} v\right|^{2}\right) \mathrm{d} \omega
\end{aligned}
$$

where $\partial_{\theta}:=\partial / \partial \theta, \partial_{\varphi}:=\partial / \partial \varphi, \partial_{\theta \theta}=\partial_{\theta} \partial_{\theta}$ etc. Note that in this setting $\|\tilde{v}\|_{0, \tilde{\Omega}}=|[v]|_{0, \Omega},|\tilde{v}|_{1, \tilde{\Omega}}=[v]_{1, \Omega}$, but $|\tilde{v}|_{2, \tilde{\Omega}} \neq[v]_{2, \Omega}$, only $|\tilde{v}|_{2, \tilde{\Omega}} \gtrsim[v]_{2, \Omega}$. This is, however, sufficient for our purposes.

The usual norm symbols are used in connection with the parameter domain $\Omega$ in Section 4.3

$$
\|v\|_{k, \Omega}^{2}:=\sum_{j=0}^{k}|v|_{j, \Omega}^{2}, \quad|v|_{k, \Omega}^{2}:=\sum_{i+j=k} \int_{\Omega}\left|\partial_{\varphi}^{i} \partial_{\theta}^{j} v\right|^{2} \mathrm{~d} \theta \mathrm{d} \varphi .
$$

The same symbols, $\|\cdot\|$ and $|[\cdot]|$, are used for the norms/seminorms of vector functions $v=\left(v_{1}, v_{2}, v_{3}\right)^{\top}$. The square of the (semi-)norm of a vector function is defined as the sum of the squares of the (semi-)norms of its components.

By $H_{0}^{1}(\tilde{\Omega})$ we denote the space of $H^{1}(\tilde{\Omega})$ functions vanishing on $\tilde{\Gamma}=\partial \tilde{\Omega}$. Furthermore, we define the spaces $H:=L^{2}(\tilde{\Omega})^{3}$ and $V:=H_{0}^{1}(\tilde{\Omega})^{3}$ of complex vector functions and identify them with the corresponding spaces $L^{2}(\Omega)^{3}$ and $H_{0}^{1}(\Omega)^{3}$, respectively. These spaces are equipped with the norms

$$
\|v\|_{H}:=|[v]|_{0, \Omega}, \quad\|v\|_{V}:=\left(|[v]|_{0, \Omega}^{2}+\frac{1}{4}[v]_{1, \Omega}^{2}\right)^{1 / 2}
$$

The factor $\frac{1}{4}$ is used in order to underline that the constants $M_{1}$ and $M_{2}$ in Lemma 2.1 below are just the constants in relation (6).

The weak formulation of problem (12) is derived, e.g., in [27] by inserting the ansatz (11) into (9). For presenting the result, we introduce some abbreviating notation. We define for $u \in H_{0}^{1}(\Omega)$

$$
e_{j}(u):=-\frac{1}{2} A_{j} u+B_{j} \partial_{\theta} u+C_{j} \partial_{\varphi} u, \quad s_{j}(u):=A_{j} u, \quad j=1,2,3,
$$

where

$$
\begin{aligned}
& A_{1}:=\cos \varphi \sin \theta, B_{1}:=\cos \varphi \cos \theta, C_{1}:=-\sin \varphi / \sin \theta, \\
& A_{2}:=\sin \varphi \sin \theta, B_{2}:=\sin \varphi \cos \theta, C_{2}:=\cos \varphi / \sin \theta, \\
& A_{3}:=\cos \theta, \quad B_{3}:=-\sin \theta, \quad C_{3}:=0
\end{aligned}
$$

The corresponding vector functions $A, B$, and $C$ arise in the representation of the nabla operator $\left(\partial_{1}, \partial_{2}, \partial_{3}\right)^{\top}$ in spherical coordinates after setting $r=1$. Now we introduce the mappings $k: V \times V \rightarrow \mathbb{C}, m: H \times H \rightarrow \mathbb{C}$, 
$d: H \times V \rightarrow \mathbb{C}$, and $g: V \times V \rightarrow \mathbb{C}$

$$
\begin{aligned}
k(u, v) & :=\sum_{i, j, l, n=1}^{3} \int_{\Omega} a_{i j l n} e_{j}\left(u_{i}\right) e_{n}\left(\bar{v}_{l}\right) \mathrm{d} \omega, \\
m(u, v) & :=\sum_{i, j, l, n=1}^{3} \int_{\Omega} a_{i j l n} s_{j}\left(u_{i}\right) s_{n}\left(\bar{v}_{l}\right) \mathrm{d} \omega \\
d(u, v) & :=\sum_{i, j, l, n=1}^{3} \int_{\Omega} a_{i j l n} s_{j}\left(u_{i}\right) e_{n}\left(\bar{v}_{l}\right) \mathrm{d} \omega, \\
g(u, v) & :=\overline{d(v, u)}-d(u, v) .
\end{aligned}
$$

The eigenvalue problem reads: Find $\alpha \in \mathbb{C}$ and $u \in V \backslash\{0\}$ such that

$$
0=\alpha(\alpha+1) m(u, v)-(\alpha+1) d(u, v)+\alpha \overline{d(v, u)}+\frac{1}{4} m(u, v)+\frac{1}{2} d(u, v)+\frac{1}{2} \overline{d(v, u)}-k(u, v) \quad \forall v \in V
$$

Since the eigenvalues $\alpha$ of the generalized eigenvalue problem (12) are distributed symmetrically with respect to the line $\operatorname{Re} \alpha=-1 / 2$ (we will see this in Th. 3.9, p. 1054), we introduce the new parameter

$$
\lambda=\alpha+1 / 2
$$

Then the weak formulation of the transformed eigenvalue problem is much simpler and reads: Find $\lambda \in \mathbb{C}$ and $u \in V \backslash\{0\}$ such that

$$
k(u, v)=\lambda g(u, v)+\lambda^{2} m(u, v) \quad \forall v \in V
$$

The number $\lambda$ is called eigenvalue of problem (14), and the vector function $u$ is called eigenelement corresponding to $\lambda$.

Lemma 2.1. The sesquilinear forms $k$ and $m$ are Hermitean, the form $g$ is skew-Hermitean,

$$
\begin{array}{ll}
k(u, v)=\overline{k(v, u)} & \forall u, v \in V, \\
g(u, v)=-\overline{g(v, u)} & \forall u, v \in V, \\
m(u, v)=\overline{m(v, u)} & \forall u, v \in H .
\end{array}
$$

Moreover, the ellipticity and boundedness properties

$$
\begin{array}{rlrl}
M_{1}\|u\|_{V}^{2} \leq k(u, u) & \leq M_{2}\|u\|_{V}^{2} & & \forall u \in V, \\
M_{1}\|u\|_{H}^{2} \leq m(u, u) & \leq M_{2}\|u\|_{H}^{2} & \forall u \in H, \\
|g(u, u)| \leq 2 \sqrt{k(u, u)} \sqrt{m(u, u)} & \forall u \in V, \\
|d(u, v)| \leq \sqrt{m(u, u)} \sqrt{k(v, v)} & & \forall u \in H, v \in V,
\end{array}
$$

hold.

Proof. The properties follow from the definitions of the sesquilinear forms, the symmetry assumptions (5) and the ellipticity and boundedness assumptions (6) on the coefficients $a_{i j l n}, i, j, l, n=1,2,3$. 


\section{Two RELATED EIGENVALUE PROBLEMS IN INFINITE DIMENSIONAL SPACES}

\subsection{Notation for operator eigenvalue problems}

In this section we recall notation for general eigenvalue problems which will be used in two different cases in Sections 3.2 and 3.3 .

Let $\mathcal{A}(\alpha): X \rightarrow Y$ be an operator pencil (operator function) mapping, for fixed $\alpha \in \mathbb{C}$, the Banach space $\mathrm{X}$ into the Banach space $\mathrm{Y}$. Thus we have $\mathcal{A}: \mathbb{C} \rightarrow \mathcal{L}(X, Y)$. In this paper we specifically assume that $\mathcal{A}$ is quadratic in $\alpha$. A set

$$
\sigma(\mathcal{A}) \subset \mathbb{C}
$$

is called the spectrum of $\mathcal{A}(\cdot)$ if for $\alpha \in \sigma(\mathcal{A})$ there is no bounded inverse operator $(\mathcal{A}(\alpha))^{-1}: Y \rightarrow X$. The number $\alpha_{0}$ is called eigenvalue of $\mathcal{A}(\cdot)$, if there exists an element $u\left(\alpha_{0} ; \cdot\right) \in X \backslash\{0\}$ such that $\mathcal{A}\left(\alpha_{0}\right) u\left(\alpha_{0} ; \cdot\right)=0$. This element $u\left(\alpha_{0} ; \cdot\right)$ is called eigenelement of the operator pencil $\mathcal{A}(\cdot)$ corresponding to $\alpha_{0}$. Shortly, we say $\left(\alpha_{0}, u\left(\alpha_{0} ; \cdot\right)\right)$ is an eigenpair. The set

$$
U\left(\mathcal{A}, \alpha_{0}\right):=\left\{u\left(\alpha_{0} ; \cdot\right): \mathcal{A}\left(\alpha_{0}\right) u\left(\alpha_{0} ; \cdot\right)=0\right\} \cup\{0\}
$$

is a closed subspace in $X$, which is called the eigensubspace of the operator pencil $\mathcal{A}(\cdot)$ corresponding to $\alpha_{0}$. The dimension

$$
n\left(\mathcal{A}, \alpha_{0}\right):=\operatorname{dim} U\left(\mathcal{A}, \alpha_{0}\right)
$$

of this subspace is called the geometric multiplicity of the eigenvalue $\alpha_{0}$.

A set of elements $u^{0}, u^{1}, \ldots, u^{k-1}$, is called Jordan chain of the length $k$ of $\mathcal{A}(\cdot)$ at $\alpha_{0}$ if the following relations hold:

$$
\begin{aligned}
\mathcal{A}\left(\alpha_{0}\right) u^{0} & =0 \\
\mathcal{A}\left(\alpha_{0}\right) u^{1}+\mathcal{A}^{\prime}\left(\alpha_{0}\right) u^{0} & =0 \\
\mathcal{A}\left(\alpha_{0}\right) u^{i}+\mathcal{A}^{\prime}\left(\alpha_{0}\right) u^{i-1}+\frac{1}{2} \mathcal{A}^{\prime \prime}\left(\alpha_{0}\right) u^{i-2} & =0, \quad i=2,3, \ldots, k-1 .
\end{aligned}
$$

The elements $u^{0}=u^{0}\left(\alpha_{0} ; \cdot\right), u^{1}=u^{1}\left(\alpha_{0} ; \cdot\right), \ldots, u^{k-1}=u^{k-1}\left(\alpha_{0} ; \cdot\right)$, of any Jordan chain of the operator pencil $\mathcal{A}(\cdot)$ at $\alpha_{0}$ are called generalized eigenelements corresponding to $\alpha_{0}$. The closed linear hull of all the generalized eigenelements of $\mathcal{A}(\cdot)$ at $\alpha_{0}$ is called generalized eigensubspace

$$
W\left(\mathcal{A}, \alpha_{0}\right)
$$

of $\mathcal{A}(\cdot)$ at $\alpha_{0}$. The maximal length of Jordan chains beginning with $u^{0} \in U\left(\mathcal{A}, \alpha_{0}\right)$ is called order

$$
\nu\left(\mathcal{A}, u^{0}\right)
$$

of the eigenelement $u^{0}$. Different eigenelements to the same eigenvalue $\alpha_{0}$ may have different orders, therefore we introduce by

$$
\varkappa\left(\mathcal{A}, \alpha_{0}\right)=\max _{u \in U\left(\mathcal{A}, \alpha_{0}\right) \backslash\{0\}} \nu(\mathcal{A}, u)
$$

the maximal order of generalized eigenelements corresponding to $\alpha_{0}$.

A system of eigenelements $u_{1}^{0}, u_{2}^{0}, \ldots, u_{l}^{0}$, from $U\left(\mathcal{A}, \alpha_{0}\right)$ is a canonical basis of eigenelements of $\mathcal{A}(\cdot)$ at $\alpha_{0}$ if

$$
\nu\left(\mathcal{A}, u_{1}^{0}\right)=\varkappa\left(\mathcal{A}, \alpha_{0}\right)
$$


and $u_{i}^{0}, 2 \leq i \leq l$, is an eigenelement of the maximal possible order belonging to some direct complement $\mathcal{M}_{i}$ in $U\left(\mathcal{A}, \alpha_{0}\right)$ to the linear hull $\mathcal{L}\left(u_{1}^{0}, \ldots, u_{i-1}^{0}\right)$ of the eigenelements $u_{1}^{0}, \ldots, u_{i-1}^{0}$, i.e.

$$
\nu\left(\mathcal{A}, u_{i}^{0}\right)=\max _{u \in \mathcal{M}_{i} \backslash\{0\}} \nu(\mathcal{A}, u), \quad i=2,3, \ldots, l .
$$

The vector $\left(\nu_{1}, \nu_{2}, \ldots, \nu_{l}\right)$ with $\nu_{i}=\nu\left(\mathcal{A}, u_{i}^{0}\right), i=1,2, \ldots, l$, is the same for every canonical basis of eigenelements. The number

$$
\mu\left(\mathcal{A}, \alpha_{0}\right)=\nu_{1}+\nu_{2}+\ldots+\nu_{l}
$$

is called algebraic multiplicity of the eigenvalue $\alpha_{0}$. If the algebraic multiplicity of an eigenvalue $\alpha_{0}$ exceeds it geometric multiplicity, the eigenvalue $\alpha_{0}$ is called defective. In the other case, where no generalized eigenelements exist, it is called non-defective.

Remark 3.1. Consider generalized eigenelements $u^{0}=u^{0}\left(\alpha_{0} ; \cdot\right), u^{1}=u^{1}\left(\alpha_{0} ; \cdot\right), \ldots, u^{k-1}=u^{k-1}\left(\alpha_{0} ; \cdot\right)$ of $\mathcal{A}_{c}\left(\right.$.) at $\alpha_{0}$. They are also generalized eigenelements of the weakly formulated eigenvalue problem (14) to some eigenvalue $\lambda_{0}=\alpha_{0}+\frac{1}{2}$ and satisfy the following variational equations,

$$
\begin{array}{rlrl}
k\left(u^{0}, v\right)-\lambda_{0} g\left(u^{0}, v\right)-\lambda_{0}^{2} m\left(u^{0}, v\right) & =0 & \forall v \in V, \\
k\left(u^{1}, v\right)-\lambda_{0} g\left(u^{1}, v\right)-\lambda_{0}^{2} m\left(u^{1}, v\right) & =g\left(u^{0}, v\right)+2 \lambda_{0} m\left(u^{0}, v\right) & \forall v \in V, \\
k\left(u^{i}, v\right)-\lambda_{0} g\left(u^{i}, v\right)-\lambda_{0}^{2} m\left(u^{i}, v\right) & =g\left(u^{i-1}, v\right)+2 \lambda_{0} m\left(u^{i-1}, v\right)+m\left(u^{i-2}, v\right) & \forall v \in V, \\
i=2,3, \ldots, k-1 .
\end{array}
$$

\subsection{Regularity of the eigenfunctions of the operator pencil $\mathcal{A}_{c}(\alpha)$}

First, we consider the operator pencil

$$
\mathcal{A}_{c}(\alpha): H_{0}^{1}(\tilde{\Omega})^{3} \rightarrow H^{-1}(\tilde{\Omega})^{3}
$$

defined by (12). Let us assume that $\tilde{\Omega} \subset S^{2}$ is a smooth domain. The function $\tilde{u}(\alpha ; \cdot)$ is solution of the eigenvalue problem (12),

$$
\mathcal{A}_{c}(\alpha) \tilde{u}(\alpha ; \cdot)=0 \quad \text { in } \tilde{\Omega}
$$

For every fixed $\alpha$ this equation describes an elliptic boundary value problem [23] (p. 98). Therefore one can conclude: if a nontrivial eigensolution $\tilde{u}^{0}(\alpha ; \cdot)=\tilde{u}^{0}\left(\lambda-\frac{1}{2} ; \cdot\right) \in X=H_{0}^{1}(\tilde{\Omega})^{3}$ of $\mathcal{A}_{c}(\cdot)$ exists, then it belongs to $H^{2}(\tilde{\Omega})^{3}$ and

$$
[u(\alpha ; \cdot)]_{2, \Omega} \lesssim|\tilde{u}(\alpha ; \cdot)|_{2, \tilde{\Omega}}<\infty
$$

The regularity of generalized eigenfunctions, given by the relation (15) can be derived successively: Since $\tilde{u}^{0}=\tilde{u}(\alpha ; \cdot) \in H^{2}(\tilde{\Omega})^{3}$ it follows from the second equation $\mathcal{A}_{c}(\alpha) \tilde{u}^{1}=-\mathcal{A}_{c}^{\prime}(\alpha) \tilde{u}^{0} \in L_{2}(\tilde{\Omega})^{3}$ and therefore $\tilde{u}^{1}=\tilde{u}^{1}(\alpha, \cdot) \in H_{0}^{1}(\tilde{\Omega})^{3}$ belongs to $H^{2}(\tilde{\Omega})^{3}$. The third equation implies $\tilde{u}^{2}(\alpha, \cdot) \in H^{2}(\tilde{\Omega})^{3}$. Continuing this procedure we get:

Lemma 3.2. If $\tilde{\Omega} \subset S^{2}$ is a smooth domain then the generalized eigenelements $\tilde{u}^{i}(\alpha, \cdot) \in H_{0}^{1}(\tilde{\Omega})^{3}, i=$ $0,1, \ldots, k-1$, are contained in $H^{2}(\tilde{\Omega})^{3}$. 


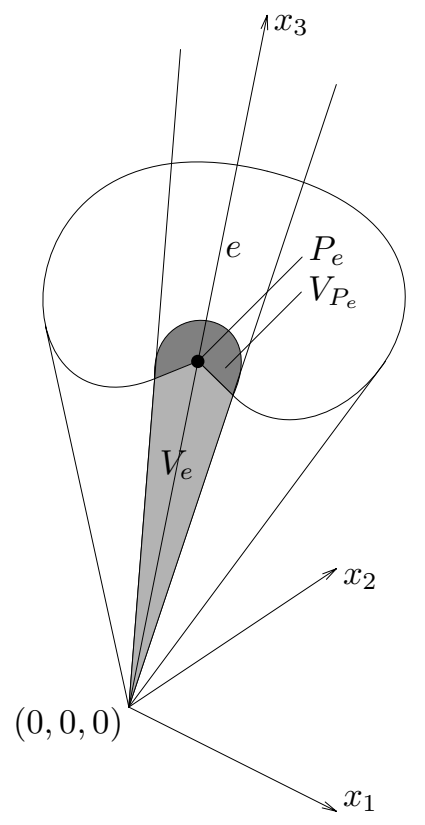

Figure 1. Vertex with one edge.

In the case of a polyhedral vertex, the operator pencil $\mathcal{A}_{c}(\alpha)$ is also defined on $H_{0}^{1}(\tilde{\Omega})^{3}$, see $(17)$. Since $\tilde{\Omega}$ has corner points (generated by the edges of the domain $G$ ), we cannot conclude in general that the generalized eigenelements $u^{i}(\alpha ; \cdot) \in H^{2}(\tilde{\Omega})^{3}$ and we have to analyze the influence of the corners on the asymptotic behaviour carefully. We follow here a paper of Dauge [11], where the corner-edge asymptotics is studied. The main idea is to introduce special fitted spherical coordinates which allow to couple the edge and corner singularities easily. We underline, that these special coordinates have auxiliary character and that the final results are independent of them.

Since singularities are of local nature we can assume, without loss of generality, that $\partial \tilde{\Omega}$ is smooth, except in one angular point $P_{e}=(0,0,1)$. Thus, the infinite cone $K$, see $(10)$, with the vertex $c=(0,0,0)$ has one edge $e$ only which coincides with the half-line $\{(0,0, z), z>0\}$. Besides the Cartesian coordinates $x=\left(x_{1}, x_{2}, x_{3}\right)$ we introduce cylindrical coordinates $(\varrho, \varphi, z)$ with $\varrho=\left(x_{1}^{2}+x_{2}^{2}\right)^{1 / 2}$. We assume that in a conical neighborhood $V_{e}$ of the edge $e$ the cone $K$ coincides with a wedge $W$ (see Fig. 1),

$$
W=\left\{(\varrho, \varphi, z): \varrho>0,0<\varphi<\omega_{0}, z \in \mathbb{R}\right\}
$$

Now, we introduce special spherical coordinates $(r, X)$, where $r=\left(x_{1}^{2}+x_{2}^{2}+x_{3}^{2}\right)^{1 / 2}$ and $X$ denotes a coordinate system at the sphere $S^{2}$ such that $X=\left(X_{1}, X_{2}\right)$ with $X_{1}=x_{1} / r=\cos \varphi \sin \theta$ and $X_{2}=x_{2} / r=\sin \varphi \sin \theta$ locally in $V_{P_{e}}=V_{e} \cap S^{2}$. Note that in $V_{P_{e}}$

$$
\operatorname{dist}\left(P_{e},\left(X_{1}, X_{2}\right)\right)=\left(X_{1}^{2}+X_{2}^{2}\right)^{1 / 2}=\frac{\varrho}{r}=\sin \theta=: R_{e}
$$

We adapt the definition of the vertex-operator pencil (12) to the new spherical coordinates and introduce an edgeoperator correspondingly. To this aim we write the linear elasticity operator $L$, introduced in Section 2, as

$$
L=L\left(\partial_{1}, \partial_{2}, \partial_{3}\right)=r^{-2} \mathcal{L}_{c}\left(X_{1}, X_{2}, r \partial r, \partial_{X_{1}}, \partial_{X_{2}}\right)
$$


Applying the Mellin transformation with respect to $r$ we get the vertex-operator pencil $\mathcal{A}_{c}\left(\alpha_{c}\right): H_{0}^{1}(\tilde{\Omega})^{3} \rightarrow$ $H^{-1}(\tilde{\Omega})^{3}$,

$$
\mathcal{A}_{c}\left(\alpha_{c}\right):=\mathcal{L}_{c}\left(X_{1}, X_{2}, \alpha_{c}, \partial_{X_{1}}, \partial_{X_{2}}\right)
$$

We denote by $\mathcal{C}_{\left[-\frac{1}{2}, \frac{1}{2}\right)}$ the set of eigenvalues of $\mathcal{A}_{c}(\cdot)$ in the strip $-\frac{1}{2} \leq \operatorname{Re} \alpha_{c}<\frac{1}{2}$ and by $u_{c}\left(\alpha_{c} ; \cdot\right)$ the corresponding eigensolutions.

The edge singularities are generated by the non-tangential (non-tangential to the edge) part of the operator $L$. Thus we remove the derivatives $\partial_{3}$ in $L$ and define

$$
L_{e}\left(\partial_{1}, \partial_{2}\right):=L\left(\partial_{1}, \partial_{2}, 0\right)
$$

Writing this operator in polar coordinates $(\varrho, \varphi)$ we have

$$
L_{e}\left(\partial_{1}, \partial_{2}\right)=\varrho^{-2} \mathcal{L}_{e}\left(\varphi, \varrho \partial_{\varrho}, \partial_{\varphi}\right)
$$

and after the Mellin transform with respect to $\varrho$

$$
\mathcal{A}_{e}\left(\alpha_{e}\right):=\mathcal{L}_{e}\left(\varphi, \alpha_{e}, \partial_{\varphi}\right)
$$

Let be $\mathcal{E}_{[0,1)}$ the set of eigenvalues of $\mathcal{A}_{e}(\cdot)$ in the strip $0 \leq \operatorname{Re} \alpha_{e}<1$ and $\Phi_{e}\left(\alpha_{e} ; \cdot\right)$ the corresponding eigensolutions.

Now we are in position to formulate the regularity results for the eigenfunctions $u_{c}\left(\alpha_{c} ; \cdot\right)$.

Theorem $3.3[10,36,40,41]$. Assume for simplicity that the eigenvalues in $\mathcal{C}_{\left[-\frac{1}{2}, \frac{1}{2}\right)}$ and $\mathcal{E}_{[0,1)}$ are non-defective. Then the weak solution $U \in H_{0}^{1}(G)^{3}$ of (9) admits the following decomposition in the vicinity of the vertex c:

$$
U=\sum_{\alpha_{c} \in \mathcal{C}_{\left[-\frac{1}{2}, \frac{1}{2}\right)}} C_{\alpha_{c}} r^{\alpha_{c}} u_{c}\left(\alpha_{c} ; X_{1}, X_{2}\right)+u_{\mathrm{rem}, c}
$$

where $C_{\alpha_{c}}$ are constants,

$$
u_{c}\left(\alpha_{c} ; X_{1}, X_{2}\right)=\sum_{\alpha_{e} \in \mathcal{E}_{[0,1)}} C_{\alpha_{e}}\left(\alpha_{c}\right) R_{e}^{\alpha_{e}} \Phi_{e}\left(\alpha_{e} ; \varphi\right)+u_{\mathrm{rem}, e}\left(\alpha_{c}\right)
$$

and $u_{\mathrm{rem}, c}$ is a remainder depending on the edge singularities,

$$
u_{\mathrm{rem}, c}=\sum_{\alpha_{e} \in \mathcal{E}_{[0,1)}} d_{\alpha_{e}}\left(x_{3}, r\right) \varrho^{\alpha_{e}} \Phi_{e}\left(\alpha_{e} ; \varphi\right)+u_{\mathrm{rem}, c, e}\left(\alpha_{c}\right)
$$

Here, $R_{e}:=\sin \theta, C_{\alpha_{c}}$ and $C_{\alpha_{e}}\left(\alpha_{c}\right)$ are constants, $d_{\alpha_{e}}$ are functions, and $u_{\mathrm{rem}, e}\left(\alpha_{c}\right), u_{\mathrm{rem}, c, e}\left(\alpha_{c}\right)$ are regular remainders.

Remark 3.4. If we admitted defective eigenvalues in $\mathcal{C}_{\left[-\frac{1}{2}, \frac{1}{2}\right)}$ and $\mathcal{E}_{[0,1)}$, then there would occur Jordan chains of $\mathcal{A}_{c}$ and $\mathcal{A}_{e}$ together with logarithmic terms of $r, R_{e}$, and $\varrho$ in the expansions $(19,20)$, and $(21)[8,35,37,40]$.

Consider now a polyhedral cone $K$, see (10). We denote by $\mathcal{E}$ the set of edges for which the set of eigenvalues $\mathcal{E}_{[0,1)}$ is not empty. Since corner and edge singularities are of local nature we see from (20) that the eigenfunctions $u_{c}$ behave like $R_{e}^{\alpha_{e}}$ in the neighborhood $\tilde{N}_{e}$ of a corner $P_{e}$ of $\tilde{\Omega}$ for $e \in \mathcal{E}$. Here, $\alpha_{e}$ is the minimal element in the 
corresponding set $\mathcal{E}_{[0,1)}$. In the parameter domain we introduce the weighted Sobolev space $V_{\beta}^{2}(\Omega), \beta=\left(\beta_{e}\right)_{e \in \mathcal{E}}$, via the norm $\|\cdot\| \|_{2, \beta, \Omega}$ which locally in $N_{e}\left(N_{e} \subset \Omega\right.$ corresponds to $\left.\tilde{N}_{e} \subset \tilde{\Omega}\right)$ is given by

$$
\begin{aligned}
\|v\|_{2, \beta_{e}, N_{e}} & \sim\left[R_{e}^{\beta_{e}} v\right]_{2, N_{e}}, \quad \text { for all } e \in \mathcal{E}, \\
\|\| v \|_{2, \beta_{e}, \Omega \backslash \bigcup_{e \in \mathcal{E}} N_{e}} & \sim|[v]|_{2, \Omega \backslash \bigcup_{e \in \mathcal{E}} N_{e}}
\end{aligned}
$$

where $\beta_{e} \in\left(1-\operatorname{Re} \alpha_{e}, 1\right)$ is arbitrary.

Corollary 3.5. The eigenfunction $u_{c}\left(\alpha_{c} ; \cdot\right)$ with respect to some eigenvalue $\alpha_{c} \in \mathcal{C}_{\left[-\frac{1}{2}, \frac{1}{2}\right)}$ of $\mathcal{A}_{c}(\cdot)$ belongs to $V_{\beta}^{2}(\Omega)^{3}$, with entries $\beta_{e} \in\left(1-\operatorname{Re} \alpha_{e}, 1\right)$ of $\beta$. That means, the corresponding norm is bounded, $\left\|u_{c}\right\|_{2, \beta, \Omega}<\infty$.

Proof. Let $P_{e}$ be a corner which is described in the parameter plane by $\left(\varphi_{0}, \theta_{0}\right)$. Assume first that $\theta_{0} \notin\{0, \pi\}$. From (20) we find that the exponent of $R_{e}$ of the expansion of $R_{e}^{\beta_{e}} u_{c}$ is $\beta_{e}+\operatorname{Re} \alpha_{e}>1$, therefore

$$
\left[R_{e}^{\beta_{e}} u_{c}\right]_{2, N_{e}} \lesssim\left|R_{e}^{\beta_{e}} \tilde{u}_{c}\right|_{2, \tilde{N}_{e}}<\infty
$$

If $\theta_{0}=0$ then $R_{e}=\sin \theta \sim \theta$ and we obtain

$$
\left[\theta^{\beta_{e}} u_{c}\right]_{2, N_{e}}<\infty
$$

by direct calculation. The case $\theta_{0}=\pi$ can be treated analogously by using $R_{e}=\sin (\pi-\theta)$.

We end this section by citing some results about the distribution of the eigenvalues which are of interest in the context of our work.

Lemma 3.6. Let be $K=\left\{\left(x_{1}, x_{2}, x_{3}\right): x_{3}>f\left(x_{1}, x_{2}\right)\right\}$ where $f$ is a continuous and positively homogeneous function of degree 1, i.e., $f\left(a x_{1}, a x_{2}\right)=a f\left(x_{1}, x_{2}\right)$ for all $a>0$.

(i) Then the strip $\left|\operatorname{Re} \alpha_{c}+\frac{1}{2}\right|<\frac{1}{2}$ does not contain eigenvalues of the pencil $\mathcal{A}_{c}\left(\alpha_{c}\right)$ [24] (Th. 11.2.2, p. 353).

(ii) If the restriction of $f$ to the sphere $S^{1}=\left\{\left(x_{1}, x_{2}\right): x_{1}^{2}+x_{2}^{2}=1\right\}$ belongs to the Sobolev space $H^{1}\left(S^{1}\right)$, then the strip $\left|\operatorname{Re} \alpha_{c}+\frac{1}{2}\right| \leq \frac{1}{2}$ has no eigenvalues of the pencil $\mathcal{A}_{c}\left(\alpha_{c}\right)$ [24] (Th. 11.3.3, p. 364).

(iii) If $N$ edges with the opening angles $\varphi_{j}, j=1 \ldots N$, meet in the vertex $c, \varphi_{j} \in(0, \pi)$ for $j=2, \ldots, N$ and $\varphi_{1} \in(0,2 \pi]$, then the strip $\left|\operatorname{Re} \alpha_{c}+\frac{1}{2}\right| \leq 1$ is free of eigenvalues of the pencil $\mathcal{A}_{c}\left(\alpha_{c}\right)$ [24] (Th. 11.4.1, p. 367).

There are also results on the distribution of the eigenvalues of the pencil $\mathcal{A}_{e}\left(\alpha_{e}\right)$.

Lemma 3.7 [24] (Th. 8.6.2, p. 286, and Th. 11.2.2, p. 353). Let be

$$
K=\left\{\left(x_{1}, x_{2}\right): 0<\left(x_{1}^{2}+x_{2}^{2}\right)^{1 / 2}<\infty, 0<\arctan \frac{x_{2}}{x_{1}}<\omega_{0}\right\}
$$

(i) If $\omega_{0} \in(0, \pi)$, then the strip $\left|\operatorname{Re} \alpha_{e}\right| \leq 1$ does not contain eigenvalues of the pencil $\mathcal{A}_{e}\left(\alpha_{e}\right)$ [24] (Th. 8.6.2, p. 286).

(ii) If $\omega_{0} \in(\pi, 2 \pi)$, then the strip $0<\operatorname{Re} \alpha_{e} \leq 1$ contains exactly 2 eigenvalues (counting multiplicity) of the pencil $\mathcal{A}_{e}\left(\alpha_{e}\right)$ [24] (Th. 8.6.2, p. 286).

(iii) If $\omega_{0}=\pi$ or $\omega_{0}=2 \pi$, then in the half plane $\operatorname{Re} \alpha_{e}>0$ the only eigenvalues $\alpha_{e, k}=k$ or $\alpha_{e, k}=\frac{k}{2}$, respectively, occur with two linearly independent eigensolutions (no associated eigenfunctions exist) [24] (Sect. 8.6.2, p. 284f).

(iv) The strip $\left|\operatorname{Re} \alpha_{e}\right|<\frac{1}{2}$ is free of eigenvalues of the pencil $\mathcal{A}_{e}\left(\alpha_{e}\right)$ [24] (Th. 11.2.2, p. 353). 


\subsection{Regularity of the eigenfunctions of the operator pencil $\mathcal{B}(\lambda)$}

In the previous section we reviewed regularity results for the eigenfunctions of the operator $\mathcal{A}_{c}(\alpha): V \rightarrow V^{\prime}$, $V=H_{0}^{1}(\Omega)^{3}$ which is the typical setting in the corresponding literature. In this section we describe properties of a related operator pencil $\mathcal{B}(\lambda): V \rightarrow V$ and its adjoint which we will apply to formulate general spectral approximation results in the next section.

We come back to the weak eigenvalue problem (14) which is basic for the discretization. We introduce the operators $\mathcal{M}: V \rightarrow V$ and $\mathcal{D}: V \rightarrow V$ by the relations

$$
\begin{aligned}
& \mathcal{M} u \in V, k(\mathcal{M} u, v)=m(u, v) \forall v \in V, \\
& \mathcal{D} u \in V, \quad k(\mathcal{D} u, v)=d(u, v) \quad \forall v \in V .
\end{aligned}
$$

The adjoint operator $\mathcal{D}^{*}: V \rightarrow V$ is defined by the equality

$$
k(\mathcal{D} u, v)=k\left(u, \mathcal{D}^{*} v\right) \quad \forall u, v \in V .
$$

We set $\mathcal{G}=\mathcal{D}^{*}-\mathcal{D}$ and introduce the operator pencil $\mathcal{B}(\cdot): \mathbb{C} \rightarrow \mathcal{L}(V, V)$ by

$$
\mathcal{B}(\lambda)=I-\lambda \mathcal{G}-\lambda^{2} \mathcal{M}, \quad \lambda \in \mathbb{C} .
$$

The variational eigenvalue problem (14) is then equivalent to the eigenvalue problem for the operator pencil $\mathcal{B}(\cdot)$ : Find $\lambda \in \mathbb{C}, u \in V \backslash\{0\}$, such that

$$
\mathcal{B}(\lambda) u=0
$$

Note that the definition of $\mathcal{B}(\lambda)$ is equivalent to $\mathcal{B}(\lambda)=J^{-1} \mathcal{A}_{c}(\lambda-1 / 2)$, where $J$ is the isometry from $V$ onto $V^{\prime}$ defined by $k(u, v)=\langle J u, v\rangle_{V^{\prime}, V} \quad \forall u, v \in V$.

Moreover, we define the operators $\mathcal{G}^{*}$ and $\mathcal{M}^{*}$ analogously to $\mathcal{D}^{*}$ and introduce the operator pencil $\mathcal{B}^{*}(\cdot)$ by the equality

$$
\mathcal{B}^{*}(\lambda)=[\mathcal{B}(\lambda)]^{*}, \quad \lambda \in \mathbb{C}
$$

Now, we investigate the spectral properties of the operator pencil $\mathcal{B}(\cdot)$ on the basis of classical results from the spectral theory of operator pencils, see, for example, [25,26,31,48]. First, from Lemma 2.1 and the compact embedding $V \stackrel{\text { c }}{\hookrightarrow} H$, where $H=L^{2}(\Omega)^{3}$, see Section 2.2 , we obtain the following lemma which then implies Theorem 3.9.

Lemma 3.8. The operators $\mathcal{G}: V \rightarrow V, \mathcal{M}: V \rightarrow V, \mathcal{D}: V \rightarrow V, \mathcal{D}^{*}: V \rightarrow V$, are compact and $\mathcal{M}^{*}=\mathcal{M}$ $\mathcal{G}^{*}=-\mathcal{G}$.

Theorem 3.9. The spectrum $\sigma(\mathcal{B})$ of problem (14) consists of isolated eigenvalues with finite algebraic multiplicities and with the only one possible accumulation point at infinity. If $\lambda_{0}$ is an eigenvalue of problem (14) then so are $-\lambda_{0}, \bar{\lambda}_{0}$ and $-\bar{\lambda}_{0}$.

Proof. Since $\lambda=0$ is a regular value of the operator pencil $\mathcal{B}(\lambda)=I-\lambda \mathcal{G}-\lambda^{2} \mathcal{M}, \lambda \in \mathbb{C}$, with compact operators $\mathcal{G}$ and $\mathcal{M}$, the assertions follow from $[27,31]$.

Lemma 3.10. $\mathcal{B}^{*}$ and $\mathcal{B}$ are also related by $\mathcal{B}^{*}(\lambda)=\mathcal{B}(-\bar{\lambda})$ for $\lambda \in \mathbb{C}$.

Proof. The assertion follows from the definition and the properties of $\mathcal{B}, \mathcal{G}$ and $\mathcal{M}$ :

$$
\begin{aligned}
k(\mathcal{B}(\lambda) u, v) & =k\left(\left(I-\lambda \mathcal{G}-\lambda^{2} \mathcal{M}\right) u, v\right)=k\left(u,\left(I-\bar{\lambda} \mathcal{G}^{*}-\bar{\lambda}^{2} \mathcal{M}^{*}\right) v\right) \\
& =k\left(u,\left(I-(-\bar{\lambda}) \mathcal{G}-(-\bar{\lambda})^{2} \mathcal{M}\right) v\right)=k(u, \mathcal{B}(-\bar{\lambda}) v)=k\left(u, \mathcal{B}^{*}(\lambda) v\right)
\end{aligned}
$$

for $u, v \in V, \lambda \in \mathbb{C}$. 
Now, we consider the adjoint eigenvalue problem: Find $\lambda \in \mathbb{C}, u \in V \backslash\{0\}$, such that $\mathcal{B}^{*}(\lambda) u=0$. By using Lemma 3.10, its variational form reads: Find $\lambda \in \mathbb{C}, u \in V \backslash\{0\}$, such that

$$
k(u, v)=-\bar{\lambda} g(u, v)+\bar{\lambda}^{2} m(u, v) \quad \forall v \in V .
$$

Theorem 3.11. The spectra of $\mathcal{B}$ and $\mathcal{B}^{*}$ coincide,

$$
\sigma(\mathcal{B})=\sigma\left(\mathcal{B}^{*}\right)
$$

and for any eigenvalue $\lambda_{0} \in \sigma(\mathcal{B})$ the maximal order of generalized eigenelements, $\varkappa$, the geometric multiplicity, $n$, and the algebraic multiplicity, $\mu$, are finite and equal for $\mathcal{B}$ and $\mathcal{B}^{*}$ :

$$
\begin{aligned}
& \varkappa\left(\mathcal{B}, \lambda_{0}\right)=\varkappa\left(\mathcal{B}^{*}, \lambda_{0}\right)<\infty \\
& n\left(\mathcal{B}, \lambda_{0}\right)=n\left(\mathcal{B}^{*}, \lambda_{0}\right)<\infty, \\
& \mu\left(\mathcal{B}, \lambda_{0}\right)=\mu\left(\mathcal{B}^{*}, \lambda_{0}\right)<\infty .
\end{aligned}
$$

Proof. These results follow, for example, from [17, 25, 26, 30, 32, 48].

We use now the regularity results for the Jordan chains of the operator $\mathcal{A}_{c}$ in order to clarify the smoothness of the generalized eigenfunctions of the operators $\mathcal{B}$ and $\mathcal{B}^{*}$. The definition of these generalized eigenfunctions was already given in Remark 3.1 on page 1050. Note that the operator pencils $\mathcal{A}_{c}(\alpha): V \rightarrow V^{\prime}$ and $\mathcal{B}(\lambda): V \rightarrow V$ are closely connected via $\lambda=\alpha+\frac{1}{2}$ and the Riesz representation theorem.

Lemma 3.12. The generalized eigenelements of the operator pencil $\mathcal{B}(\cdot)$ at $\lambda$ and of the operator pencil $\mathcal{B}^{*}(\cdot)$ at $-\bar{\lambda}$, respectively, belong to $V_{\beta}^{2}(\Omega)^{3}$, with entries $\beta_{e} \in\left(1-\operatorname{Re} \alpha_{e}, 1\right)$ of $\beta$. Moreover, for an eigenpair $(\lambda, u(\lambda ; \cdot))$ of $\mathcal{B}(\cdot)$ or $\mathcal{B}^{*}(\cdot)$ the estimate

$$
\| u(\lambda ; \cdot)||_{2, \beta, \Omega} \leq C\left(|\lambda|^{2}|[u(\lambda ; \cdot)]|_{0, \Omega}+|\lambda||[u(\lambda ; \cdot)]|_{1, \Omega}\right)
$$

holds with a constant $C$ independent of $\lambda$ and $u(\lambda ; \cdot)$.

Proof. For simplicity, we consider again the case of non-defective eigenvalues $\alpha_{c} \in \mathcal{C}_{\left[-\frac{1}{2}, \frac{1}{2}\right)}$ and $\alpha_{e} \in \mathcal{E}_{[0,1)}$. Setting $\alpha_{c}=\lambda_{c}-\frac{1}{2}$, formulae (19) and (20) describe the behaviour of the eigensolutions $u(\lambda ; \cdot)$ of the operator pencil $\mathcal{B}$. As in the proof of Corollary 3.5 we find that $\|u(\lambda ; \cdot)\| \|_{2, \beta, \Omega}<\infty$. Using the definition of the generalized eigenfunctions, see Remark 3.1, recurrently, we get analogous results for the generalized eigenfunctions.

Now we pass to the estimate $(23)$. For a fixed eigenpair $(\lambda, u(\lambda ; \cdot))$ we have

$$
k(u(\lambda ; \cdot), v(\cdot))=\lambda g(u(\lambda ; \cdot), v(\cdot))+\lambda^{2} m(u(\lambda ; \cdot), v(\cdot)) \quad \forall v \in V .
$$

Since $u(\lambda ; \cdot) \in V_{\beta}^{2}(\Omega)^{3}$ for $\beta_{e} \in\left(1-\operatorname{Re} \alpha_{e}, 1\right)$ this weakly formulated $V$-elliptic boundary value problem is equivalent to a classical one,

$$
K_{2} u(\lambda ; \cdot)=\lambda K_{1} u(\lambda ; \cdot)+\lambda^{2} K_{0} u(\lambda ; \cdot) \quad \text { in } \tilde{\Omega}, \quad u(\lambda ; \cdot)=0 \quad \text { on } \partial \tilde{\Omega},
$$

where $K_{i}$ are differential operators of the order $i$. Due to the well known a priori estimates for elliptic boundary value problems in weighted spaces [22] the estimate

$$
\begin{aligned}
\left.\|u(\lambda ; \cdot)\|\right|_{2, \beta, \Omega} & \leq C\left|\left[\lambda K_{1} u(\lambda ; \cdot)+\lambda^{2} K_{0} u(\lambda ; \cdot)\right]\right|_{0, \Omega} \\
& \leq C\left(|\lambda||[u(\lambda ; \cdot)]|_{1, \Omega}+|\lambda|^{2}|[u(\lambda ; \cdot)]|_{0, \Omega}\right)
\end{aligned}
$$

follows. 
We have seen that the regularity of the eigenfunctions $u(\lambda ; \cdot)$ of $\mathcal{B}(\cdot)$ is dominated by the geometry of the spherical cone, that means, by the eigenvalues $\alpha_{e} \in \mathcal{E}_{[0,1)}$. Therefore, the arguments for the derivation of the estimate $(23)$ can be repeated for an arbitrary eigenvalue $\lambda$.

Since $\mathcal{B}^{*}(-\bar{\lambda})=\mathcal{B}(\lambda): V \rightarrow V$, the regularity results are valid for the generalized eigenelements of $\mathcal{B}^{*}$, too.

\section{The APproximate EIgenvalue Problem}

\subsection{General spectral approximation results}

In this section we review approximation results taken from the papers of Karma [19-21].

Let $V_{h} \subset V$ be given finite-dimensional subspaces such that for any $v \in V$

$$
\varepsilon_{h}(v):=\inf _{v_{h} \in V_{h}}\left\|v-v_{h}\right\|_{V} \rightarrow 0 \quad \text { for } h \rightarrow 0
$$

Problem (14) is approximated by the following finite-dimensional problem: Find $\lambda_{h} \in \mathbb{C}, u_{h} \in V_{h} \backslash\{0\}$, such that

$$
k\left(u_{h}, v_{h}\right)=\lambda_{h} g\left(u_{h}, v_{h}\right)+\lambda_{h}^{2} m\left(u_{h}, v_{h}\right) \quad \forall v_{h} \in V_{h} .
$$

The number $\lambda_{h}$ is called approximate eigenvalue and the vector function $u_{h}$ is called eigenelement corresponding to $\lambda_{h}$.

We define the projection operator $\mathcal{P}_{h}: V \rightarrow V_{h}$ by

$$
\mathcal{P}_{h} u \in V_{h}, \quad k\left(\mathcal{P}_{h} u, v_{h}\right)=k\left(u, v_{h}\right) \quad \forall v_{h} \in V_{h}, u \in V,
$$

set $\mathcal{G}_{h}=\mathcal{P}_{h} \mathcal{G}, \mathcal{M}_{h}=\mathcal{P}_{h} \mathcal{M}, \mathcal{D}_{h}=\mathcal{P}_{h} \mathcal{D}$ and introduce in analogy to Section 3.3 the operator pencil $\mathcal{B}_{h}(\cdot)$ by the formula $\mathcal{B}_{h}(\lambda)=I-\lambda \mathcal{G}_{h}-\lambda^{2} \mathcal{M}_{h}, \lambda \in \mathbb{C}$. Then the variational eigenvalue problem (25) is equivalent to: Find $\lambda_{h} \in \mathbb{C}, u_{h} \in V_{h} \backslash\{0\}$, such that

$$
\mathcal{B}_{h}\left(\lambda_{h}\right) u_{h}=0
$$

Lemma 4.1. The operator $\mathcal{P}_{h}: V \rightarrow V_{h}$ is a self-adjoint projector with

$$
\left\|u-\mathcal{P}_{h} u\right\|_{V} \leq \sqrt{\frac{M_{2}}{M_{1}}} \varepsilon_{h}(u) \quad \forall u \in V,
$$

where $M_{1}$ and $M_{2}$ are the constants from (6).

Proof. These results follow from the projection property of $\mathcal{P}_{h}$ and the properties of the sesquilinear form $k(.,$. given in Lemma 2.1.

Now, let us formulate properties of the operators $\mathcal{G}_{h}, \mathcal{M}_{h}, \mathcal{D}_{h}, \mathcal{B}_{h}(\cdot)$ analogously to the properties described in Section 3.3 for the infinite-dimensional case.

Lemma 4.2. The operators $\mathcal{G}_{h}: V_{h} \rightarrow V_{h}, \mathcal{M}_{h}: V_{h} \rightarrow V_{h}, \mathcal{D}_{h}: V_{h} \rightarrow V_{h}, \mathcal{D}_{h}^{*}: V_{h} \rightarrow V_{h}$ are compact and $\mathcal{M}_{h}^{*}=\mathcal{M}_{h}, \mathcal{G}_{h}^{*}=-\mathcal{G}_{h}$.

Lemma 4.3. $\mathcal{B}_{h}^{*}$ and $\mathcal{B}_{h}$ are related by $\mathcal{B}_{h}^{*}(\lambda)=\mathcal{B}_{h}(-\bar{\lambda})$ for $\lambda \in \mathbb{C}$.

Theorem 4.4. The spectrum $\sigma\left(\mathcal{B}_{h}\right)$ of problem (25) consists of isolated eigenvalues with finite algebraic multiplicities. If $\lambda_{h}$ is an eigenvalue of problem (25) then so are $-\lambda_{h}, \bar{\lambda}_{h}$ and $-\bar{\lambda}_{h}$. 
For $\lambda_{0} \in \sigma(\mathcal{B})$ we define

$$
\varepsilon_{h}=\max _{v \in W\left(\mathcal{B}, \lambda_{0}\right),\|v\|_{V}=1} \varepsilon_{h}(v), \quad \varepsilon_{h}^{*}=\max _{v \in W\left(\mathcal{B}^{*}, \lambda_{0}\right),\|v\|_{V}=1} \varepsilon_{h}(v)
$$

where $W\left(\mathcal{B}, \lambda_{0}\right)$ is the generalized eigensubspace, see (16).

Theorem 4.5. Let $\lambda_{0}$ be an eigenvalue of problem (14) with maximal order $\varkappa=\varkappa\left(\mathcal{B}, \lambda_{0}\right)$ of generalized eigenelements. Then there exists a sequence $\lambda_{h}$ of eigenvalues of problem (25), $\lambda_{h} \in \sigma\left(\mathcal{B}_{h}\right)$, such that $\lambda_{h} \rightarrow \lambda_{0}$ as $h \rightarrow 0$ and for sufficiently small $h$ the following error estimate is valid:

$$
\left|\lambda_{h}-\lambda_{0}\right| \lesssim\left(\varepsilon_{h} \varepsilon_{h}^{*}\right)^{1 / \varkappa}
$$

Proof. The error estimate follows from classical results [19] and Lemmata 3.8 and 4.1.

In the case $\varkappa \geq 2$ the estimate can be improved when the arithmetic mean of the approximate eigenvalues is considered.

Theorem 4.6. Let $\lambda_{0}$ be an eigenvalue of problem (14) with algebraic multiplicity $\mu=\mu\left(\mathcal{B}, \lambda_{0}\right)$. Then there exist sequences $\lambda_{i}^{h} \in \sigma\left(\mathcal{B}_{h}\right), i=1,2, \ldots, \mu$, of eigenvalues of problem (25), counted according to the algebraic multiplicity, such that $\lambda_{i}^{h} \rightarrow \lambda_{0}$ as $h \rightarrow 0$. For

$$
\hat{\lambda}_{h}=\frac{1}{\mu} \sum_{i=1}^{\mu} \lambda_{i}^{h}
$$

and sufficiently small $h$ the following error estimate is valid:

$$
\left|\hat{\lambda}_{h}-\lambda_{0}\right| \lesssim \varepsilon_{h} \varepsilon_{h}^{*}
$$

Proof. With Lemmata 3.8 and 4.1 we have proved the assumptions to apply the recent results of Karma [20,21].

Theorem 4.7. Assume that $\lambda_{0}$ is an eigenvalue of problem (14) and $\left(\lambda_{h}, u_{h}\right)$ are eigenpairs of problem (25), such that $\lambda_{h} \rightarrow \lambda_{0}$ as $h \rightarrow 0$. Then for each element $u_{h}$ there exists an eigenelement $u^{0}=u_{0}(h) \in U\left(\mathcal{B}, \lambda_{0}\right)$ of problem (14) corresponding to $\lambda_{0}$ such that for sufficiently small $h$ the following error estimate holds:

$$
\left\|u_{h}-u^{0}\right\|_{V} \lesssim \varepsilon_{h}+\left(\varepsilon_{h} \varepsilon_{h}^{*}\right)^{1 / \varkappa}
$$

Proof. The error estimate follows from [18,49], Lemmata 3.8 and 4.1 and Theorem 4.5.

\subsection{Meshes}

We define the finite element meshes in the $\varphi, \theta$-parameter plane. In view of Theorems $4.5-4.7$ the meshes must be chosen such that the eigenfunctions can be approximated well. If $\tilde{\Omega}$ is a smooth domain, the eigenfunctions are smooth and can be approximated well on standard uniform finite element meshes. In general, however, the domain $\tilde{\Omega}$ is not smooth and the mesh should be refined in the neighbourhoods of concave corners. (Due to Lem. 3.7, part (i), the eigenfunctions are regular near corners $\tilde{P}$ with interior angle $\omega_{0} \in(0, \pi)$.)

If the parameterization is such that the concave corner is not located at the north or south pole then we can use in the neighbourhood a standard graded mesh as described for example in [44]. It is characterized by (28) as stated below. In the other case, when the refinement center is one of the poles, we have to refine towards the line $\theta=0$ or $\theta=\pi$. Since a refinement in $\varphi$-direction is not necessary we arrive at the idea of using anisotropic mesh grading there, see $(29,30)$ or the illustration in Figure 4 . This anisotropy in the mesh saves many degrees of freedom and makes the analysis of the approximation error slightly more involved. 

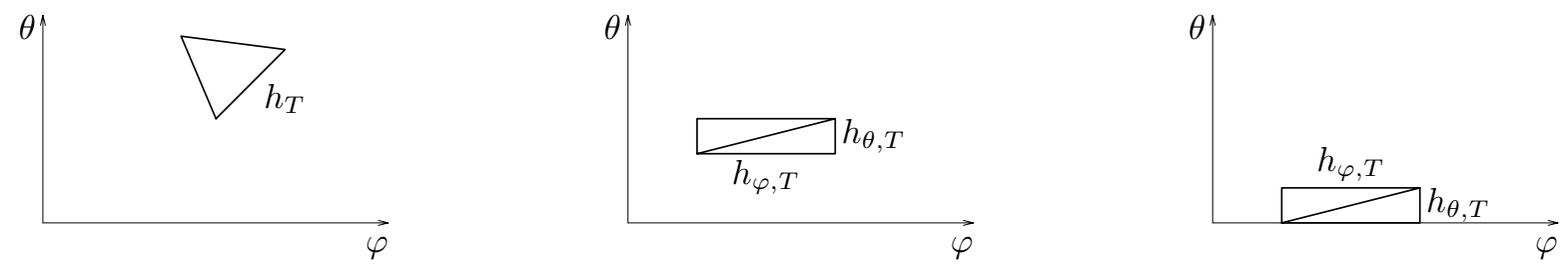

FiguRE 2. Illustration of different cases of triangles.

In order to describe the meshes we make the simplifying assumption that the domain $\tilde{\Omega} \subset S^{2}$ can be represented, by using a suitable choice of the north pole, by a polygonal domain $\Omega$ in the $(\varphi, \theta)$-plane. Then, we define a family of meshes $\mathcal{T}_{h}=\{T\}$ with the following properties. For later use we define

$$
\vartheta_{-, T}:=\inf _{(\varphi, \theta) \in T} \sin \theta, \quad \vartheta_{+, T}:=\sup _{(\varphi, \theta) \in T} \sin \theta
$$

Admissibility: We assume that $\bar{\Omega}=\bigcup_{T \in \mathcal{T}_{h}} \bar{T}$ where the elements $T$ are open triangles. Any side $E$ of any triangle $T$ is either part of the boundary $\partial \Omega$ or side of another triangle $T^{\prime} \in \mathcal{T}_{h}$.

Aspect ratio: For triangles $T$ with $\vartheta_{-, T} \geq \vartheta_{*}=$ const. $>0$ we assume that $T$ has bounded aspect ratio, without further constraints. The diameter of $T$ is denoted by $h_{T}$. For an illustration see Figure 2 , left hand side.

For $\vartheta_{-, T}<\vartheta_{*}$ we assume that two edges of $T$ are parallel to the coordinate axes. Their lengths are denoted by $h_{\varphi, T}$ and $h_{\theta, T}$ which can be chosen independently, see also Figure 2, middle and right. That means the aspect ratio of $T$ may not be bounded by a constant.

Refinement: If $\tilde{\Omega}$ is a smooth domain then $h_{T} \sim h$ for all $T \in \mathcal{T}_{h}$, and the aspect ratio of all elements is bounded.

If $\tilde{\Omega}$ is not smooth the mesh is refined in the neighbourhoods of concave corners. For each of those corners $P=\left(\varphi_{0}, \theta_{0}\right)$ the refined mesh is defined as follows where the parameter $\beta$ is the corresponding weight exponent as used in Corollary 3.5, this means $\beta_{e} \in\left(1-\operatorname{Re} \alpha_{e}, 1\right)$.

Case $1, \theta_{0} \notin\{0, \pi\}$ : The aspect ratio of the elements is bounded and

$$
h_{T} \sim \begin{cases}h^{1 /(1-\beta)} & \text { if } \operatorname{dist}(P, T)=0, \\ h[\operatorname{dist}(P, T)]^{\beta} & \text { if } \operatorname{dist}(P, T)>0 .\end{cases}
$$

This means that $h_{T} \sim h$ for $\operatorname{dist}(P, T)>C_{*}=$ const.

Case 2, $\theta_{0} \in\{0, \pi\}$ : The refinement zone is determined by $\sin \theta<\vartheta_{*}$. The elements might be anisotropic,

$$
\begin{aligned}
& h_{\varphi, T} \sim h, \\
& h_{\theta, T} \sim \begin{cases}h^{1 /(1-\beta)} & \text { if } \vartheta_{-, T}=0, \\
h \vartheta_{-, T}^{\beta} & \text { if } \vartheta_{-, T}>0 .\end{cases}
\end{aligned}
$$

The space $V_{h}$ is introduced by

$$
V_{h}=\left\{v \in V:\left.v\right|_{T} \in\left(\mathcal{P}_{1}\right)^{3} \text { for all } T \in \mathcal{T}_{h}\right\}
$$

where $\mathcal{P}_{1}$ is, as usual, the space of polynomials of maximal degree one. For estimates of the approximation error of the eigenvalues and eigenfunctions via Theorems 4.5-4.7 we need estimates of $\varepsilon_{h}$ and $\varepsilon_{h}^{*}$ from (26). Since the regularity of the functions from $W\left(\mathcal{B}, \lambda_{0}\right)$ and $W\left(\mathcal{B}^{*}, \lambda_{0}\right)$ is the same, see Lemma 3.12 , we need to estimate 
$\varepsilon_{h}(u)$ from (24) for functions $u \in V_{\beta}^{2}(\Omega)$. Therefore our aim is to bound $\left\|u-\mathrm{I}_{h} u\right\|_{V}$ where $\mathrm{I}_{h}: C(\bar{\Omega}) \rightarrow V_{h}$ is the interpolation operator with respect to the vertices of the triangulation. We start with local interpolation error estimates for scalar functions.

\subsection{Local interpolation error estimates}

The aim of this section is to estimate the interpolation error $u-\mathrm{I}_{h} u$ for scalar $u$ in the three cases which are indicated in Figure 2. Let us start with the simplest estimate.

Lemma 4.8. Let $T$ be an isotropic triangle, that means, $T$ has bounded aspect ratio, and assume that

$$
\vartheta_{-, T} \geq h_{T}
$$

Then the error estimate

$$
\left[u-\mathrm{I}_{h} u\right]_{m, T} \lesssim \vartheta_{-, T}^{-m} h_{T}^{2-m}[u]_{2, T}, \quad m=0,1
$$

holds when $|[u]|_{2, T}<\infty$.

Proof. By using the results in standard norms we get for $m=0,1$

$$
\begin{aligned}
\left|\left[\partial_{\theta}^{m}\left(u-\mathrm{I}_{h} u\right)\right]\right|_{0, T} & =\left\|\sin ^{1 / 2} \theta \partial_{\theta}^{m}\left(u-\mathrm{I}_{h} u\right)\right\|_{0, T} \\
& \leq \vartheta_{+, T}^{1 / 2}\left\|\partial_{\theta}^{m}\left(u-\mathrm{I}_{h} u\right)\right\|_{0, T} \\
& \lesssim \vartheta_{+, T}^{1 / 2} h_{T}^{2-m}|u|_{2, T} \\
& \leq \vartheta_{+, T}^{1 / 2} \vartheta_{-, T}^{-1 / 2} h_{T}^{2-m}\left(\left|\left[\sin ^{-2} \theta \partial_{\varphi \varphi} u\right]\right|_{0, T}^{2}+\left|\left[\sin ^{-1} \theta \partial_{\varphi \theta} u\right]\right|_{0, T}^{2}+\left|\left[\partial_{\theta \theta} u\right]\right|_{0, T}^{2}\right)^{1 / 2} \\
& \leq \vartheta_{+, T}^{1 / 2} \vartheta_{-, T}^{-1 / 2} h_{T}^{2-m}[u]_{2, T} .
\end{aligned}
$$

We show now that $\vartheta_{+, T} / \vartheta_{-, T} \leq 2$. Indeed, by the definition of $\vartheta_{-, T}$ and $\vartheta_{+, T}$ there exist angles $\theta_{-}$and $\theta_{+}$ with $\vartheta_{-, T}=\sin \theta_{-}, \vartheta_{+, T}=\sin \theta_{+}$, and $\left|\theta_{+}-\theta_{-}\right| \leq h_{T}$. Consequently, by using (31), we get

$$
\begin{aligned}
\vartheta_{+, T} & =\sin \theta_{+}=\sin \theta_{-} \cos \left(\theta_{+}-\theta_{-}\right)+\cos \theta_{-} \sin \left(\theta_{+}-\theta_{-}\right) \\
& =\vartheta_{-, T}\left(\cos \left(\theta_{+}-\theta_{-}\right)+\vartheta_{-, T}^{-1} \cos \theta_{-} \sin \left(\theta_{+}-\theta_{-}\right)\right) \\
& \leq 2 \vartheta_{-, T} .
\end{aligned}
$$

Analogously to (32) we show

$$
\begin{aligned}
\left|\left[\sin ^{-1} \theta \partial_{\varphi}\left(u-\mathrm{I}_{h} u\right)\right]\right|_{0, T} & =\left\|\sin ^{-1 / 2} \theta \partial_{\varphi}\left(u-\mathrm{I}_{h} u\right)\right\|_{0, T} \\
& \leq \vartheta_{-, T}^{-1 / 2}\left\|\partial_{\varphi}\left(u-\mathrm{I}_{h} u\right)\right\|_{0, T} \\
& \lesssim \vartheta_{-, T}^{-1 / 2} h_{T}|u|_{2, T} \\
& \leq \vartheta_{-, T}^{-1} h_{T}[u]_{2, T}
\end{aligned}
$$

With (32-34) we have proved the lemma.

We do not claim that this result is optimal with respect to the dependence of the right hand side on $\vartheta_{-, T}$. But this estimate is sufficient for our purposes since we use general isotropic triangles only in parts of the domain where $\vartheta_{-, T} \geq \vartheta_{*}=$ const. $>0$. 
Lemma 4.9. Let $T$ be an anisotropic triangle with two edges parallel to the coordinate axes, compare Figure 2 , middle. Assume that $|[u]|_{2, T}<\infty$. Under the assumption $\vartheta_{-, T} \gtrsim h_{\theta, T}$ the error estimates

$$
\begin{aligned}
\left|\left[u-\mathrm{I}_{h} u\right]\right|_{0, T} & \lesssim h_{\varphi, T}^{2} \vartheta_{-, T}^{2}\left|\left[\sin ^{-2} \theta \partial_{\varphi \varphi} u\right]\right|_{0, T}+h_{\varphi, T} h_{\theta, T} \vartheta_{-, T}\left|\left[\sin ^{-1} \theta \partial_{\varphi \theta} u\right]\right|_{0, T}+h_{\theta, T}^{2}\left|\left[\partial_{\theta \theta} u\right]\right|_{0, T} \\
\left|\left[\sin ^{-1} \theta \partial_{\varphi}\left(u-\mathrm{I}_{h} u\right)\right]\right|_{0, T} & \lesssim h_{\varphi, T} \vartheta_{-, T}\left|\left[\sin ^{-2} \theta \partial_{\varphi \varphi} u\right]\right|_{0, T}+h_{\theta, T}\left|\left[\sin ^{-1} \theta \partial_{\varphi \theta} u\right]\right|_{0, T} \\
\left|\left[\partial_{\theta}\left(u-\mathrm{I}_{h} u\right)\right]\right|_{0, T} & \lesssim h_{\varphi, T} \vartheta_{-, T}\left|\left[\sin ^{-1} \theta \partial_{\varphi \theta} u\right]\right|_{0, T}+h_{\theta, T}\left|\left[\partial_{\theta \theta} u\right]\right|_{0, T}
\end{aligned}
$$

hold.

Proof. The proof is analogous to that of Lemma 4.8. Only, we use the anisotropic error estimates $[2,4]$

$$
\begin{aligned}
\left\|u-\mathrm{I}_{h} u\right\|_{0, T} & \lesssim h_{\varphi, T}^{2}\left\|\partial_{\varphi \varphi} u\right\|_{0, T}+h_{\varphi, T} h_{\theta, T}\left\|\partial_{\varphi \theta} u\right\|_{0, T}+h_{\theta, T}^{2}\left\|\partial_{\theta \theta} u\right\|_{0, T}, \\
\left\|\partial_{\varphi}\left(u-\mathrm{I}_{h} u\right)\right\|_{0, T} & \lesssim h_{\varphi, T}\left\|\partial_{\varphi \varphi} u\right\|_{0, T}+h_{\theta, T}\left\|\partial_{\varphi \theta} u\right\|_{0, T} \\
\left\|\partial_{\theta}\left(u-\mathrm{I}_{h} u\right)\right\|_{0, T} & \lesssim h_{\varphi, T}\left\|\partial_{\varphi \theta} u\right\|_{0, T}+h_{\theta, T}\left\|\partial_{\theta \theta} u\right\|_{0, T}
\end{aligned}
$$

For example, the last of the three estimates is obtained as follows:

$$
\begin{aligned}
\left|\left[\partial_{\theta}\left(u-\mathrm{I}_{h} u\right)\right]\right|_{0, T} & =\left\|\sin ^{1 / 2} \theta \partial_{\theta}\left(u-\mathrm{I}_{h} u\right)\right\|_{0, T} \\
& \leq \vartheta_{+, T}^{1 / 2}\left\|\partial_{\theta}\left(u-\mathrm{I}_{h} u\right)\right\|_{0, T} \\
& \lesssim \vartheta_{+, T}^{1 / 2}\left(h_{\varphi, T}\left\|\partial_{\varphi \theta} u\right\|_{0, T}+h_{\theta, T}\left\|\partial_{\theta \theta} u\right\|_{0, T}\right) \\
& \lesssim \vartheta_{+, T}^{1 / 2}\left(h_{\varphi, T} \vartheta_{+, T}^{1 / 2}\left|\left[\sin ^{-1} \theta \partial_{\varphi \theta} u\right]\right|_{0, T}+h_{\theta, T} \vartheta_{-, T}^{-1 / 2}\left|\left[\partial_{\theta \theta} u\right]\right|_{0, T}\right) \\
& =\vartheta_{+, T}^{1 / 2} \vartheta_{-, T}^{-1 / 2}\left(h_{\varphi, T}\left(\vartheta_{+, T}^{1 / 2} \vartheta_{-, T}^{-1 / 2}\right) \vartheta_{-, T}\left|\left[\sin ^{-1} \theta \partial_{\varphi \theta} u\right]\right|_{0, T}+h_{\theta, T}\left|\left[\partial_{\theta \theta} u\right]\right|_{0, T}\right) .
\end{aligned}
$$

The arising factors $\vartheta_{+, T}^{1 / 2} \vartheta_{-, T}^{-1 / 2}$ is bounded by a constant as shown in (33).

Let $S$ be the union of all elements $T$ with $\vartheta_{-, T}=0$. If $S$ is not connected we treat all simply connected parts of $S$ separately, such that we can assume that

$$
S=\left(\varphi_{0}, \varphi_{1}\right) \times\left(0, h_{\theta, S}\right)
$$

Lemma 4.10. Assume that $|[u]|_{2, S}<\infty$. Then the interpolation operator $\mathrm{I}_{h}$ is well defined in $S$ and the estimates

$$
\left[u-\mathrm{I}_{h} u\right]_{m, S} \lesssim h_{\theta, S}^{2-m}[u]_{2, S}, \quad m=0,1
$$

hold.

Proof. By the mapping $\varphi=\hat{\varphi}, \theta=h_{\theta, S} \hat{\theta}$ we transform $S$ to $\hat{S}=\left(\varphi_{0}, \varphi_{1}\right) \times(0,1)$. On $\hat{S}$ we define the weighted Sobolev spaces

$$
\begin{aligned}
H_{1 / 2}^{k}(\hat{S}) & :=\left\{\hat{v} \in \mathcal{D}^{\prime}(\hat{S}):\|\hat{v}\|_{k ; 1 / 2, \hat{S}}<\infty\right\} \\
\|\hat{v}\|_{k ; 1 / 2, \hat{S}}^{2} & :=\sum_{j=0}^{k}|\hat{v}|_{j ; 1 / 2, \hat{S}}^{2}, \\
|\hat{v}|_{k ; 1 / 2, \hat{S}}^{2}: & =\sum_{i+j=k}\left\|\hat{\theta}^{1 / 2} \partial_{\hat{\varphi}}^{i} \partial_{\hat{\theta}}^{j} \hat{v}\right\|_{0, \hat{S}}^{2} .
\end{aligned}
$$


Such spaces were investigated by Mercier and Raugel [39] in the context of a transformation to polar/cylindrical coordinates. In particular, Theorem 4.7 of that paper states that $H_{1 / 2}^{2}(\hat{S}) \hookrightarrow C^{0}(\overline{\hat{S}})$, that means that

$$
\|\hat{u}\|_{C^{0}(\overline{\hat{S}})} \lesssim\|\hat{u}\|_{2 ; 1 / 2, \hat{S}} \leq|[\hat{u}]|_{2, \hat{S}}
$$

Consequently, $\mathrm{I}_{h}$ is well defined in $S$. Moreover,

$$
\left\|\partial_{\hat{\theta}}^{m} \hat{\mathrm{I}}_{h} \hat{u}\right\|_{0 ; 1 / 2, \hat{S}} \lesssim\|\hat{u}\|_{C^{0}(\overline{\hat{S}})}\|1\|_{0 ; 1 / 2, \hat{S}} \lesssim\|\hat{u}\|_{2 ; 1 / 2, \hat{S}} \quad m=0,1
$$

Let us now prove the interpolation error estimate. From [39] (Th. 4.6) we have the following Deny-Lions type argument,

$$
\inf _{\hat{w} \in \mathcal{P}_{1}}\|\hat{v}-\hat{w}\|_{2 ; 1 / 2, \hat{S}} \lesssim|\hat{v}|_{2 ; 1 / 2, \hat{S}}
$$

Therefore we can use the standard proof for the $\theta$-derivative of the interpolation error. By transformation to $\hat{S}$ and using $\sin \left(h_{\theta, S} \hat{\theta}\right) \sim h_{\theta, S} \hat{\theta},(37,38)$, we get for $m=0,1$,

$$
\begin{aligned}
\left|\left[\partial_{\theta}^{m}\left(u-\mathrm{I}_{h} u\right)\right]\right|_{0, S} & \lesssim h_{\theta, S}^{1-m}\left\|\partial_{\hat{\theta}}^{m}\left(\hat{u}-\hat{\mathrm{I}}_{h} \hat{u}\right)\right\|_{0 ; 1 / 2, \hat{S}} \\
& =h_{\theta, S}^{1-m} \inf _{\hat{w} \in \mathcal{P}_{1}}\left\|\partial_{\hat{\theta}}^{m}\left(\hat{u}-\hat{w}-\hat{\mathrm{I}}_{h}(\hat{u}-\hat{w})\right)\right\|_{0 ; 1 / 2, \hat{S}} \\
& \lesssim h_{\theta, S}^{1-m} \inf _{\hat{w} \in \mathcal{P}_{1}}\|\hat{u}-\hat{w}\|_{2 ; 1 / 2, \hat{S}} \\
& \lesssim h_{\theta, S}^{1-m}|\hat{u}|_{2 ; 1 / 2, \hat{S}} \\
& \lesssim h_{\theta, S}^{2-m}[u]_{2, S} .
\end{aligned}
$$

For the estimate of $\partial_{\varphi}\left(u-\mathrm{I}_{h} u\right)$ we distinguish in a first step two cases. First, if $T$ is a triangle with one side $E$ at the $\varphi$-axis then $u$ is constant on $E$ and $\partial_{\varphi} \mathrm{I}_{h} u=0$ in $T$, that means,

$$
\left|\left[\sin ^{-1} \theta \partial_{\varphi}\left(u-\mathrm{I}_{h} u\right)\right]\right|_{0, T}=\left|\left[\sin ^{-1} \theta \partial_{\varphi} u\right]\right|_{0, T}
$$

In the other case, $T$ is a triangle with one vertex at the $\varphi$-axis and one edge $E$ parallel to the $\varphi$-axis. The equivalence of norms in one-dimensional spaces, the interpolation property and the trace theorem yield that

$$
\begin{aligned}
\left|\left[\sin ^{-1} \theta \partial_{\varphi} \mathrm{I}_{h} u\right]\right|_{0, T} & \left.\sim \mid\left[\hat{\theta}^{-1} \partial_{\hat{\varphi}} \hat{\mathrm{I}}_{h} \hat{u}\right)\right]\left.\right|_{0 ; 1 / 2, \hat{T}} \\
& \sim\left|\int_{\hat{E}} \partial_{\hat{\varphi}} \hat{\mathrm{I}}_{h} \hat{u}\right|=\left|\int_{\hat{E}} \partial_{\hat{\varphi}} \hat{u}\right| \lesssim\left\|\partial_{\hat{\varphi}} \hat{u}\right\|_{1, \hat{T}} \\
& \lesssim\left\|\left.\hat{\theta}^{-1} \partial_{\hat{\varphi}} \hat{u}\right|_{0, \hat{T}}+\right\| \hat{\theta}^{-1} \partial_{\hat{\varphi} \hat{\theta}} \hat{u}\left\|_{0, \hat{T}}+\right\| \hat{\theta}^{-2} \partial_{\hat{\varphi} \hat{\varphi} \hat{u}} \|_{0, \hat{T}} \\
& \lesssim\left|\left[\sin ^{-1} \theta \partial_{\varphi} u\right]\right|_{0, T}+h_{\theta, S}\left(\left|\left[\sin ^{-1} \theta \partial_{\varphi \theta} u\right]\right|_{0, T}+\left|\left[\sin ^{-2} \theta \partial_{\varphi \varphi} u\right]\right|_{0, T}\right)
\end{aligned}
$$

Combining the two cases we obtain

$$
\left|\left[\sin ^{-1} \theta \partial_{\varphi}\left(u-\mathrm{I}_{h} u\right)\right]\right|_{0, S} \lesssim\left|\left[\sin ^{-1} \theta \partial_{\varphi} u\right]\right|_{0, S}+h_{\theta, S}\left(\left|\left[\sin ^{-1} \theta \partial_{\varphi \theta} u\right]\right|_{0, S}+\left|\left[\sin ^{-2} \theta \partial_{\varphi \varphi} u\right]\right|_{0, S}\right) .
$$


It remains to estimate the first term at the right hand side. Let $H_{0}^{1}(\hat{S}):=\left\{\hat{v} \in H^{1}(\hat{S}): \hat{v}(\hat{\varphi}, 0)=0\right\}$ and note that $\partial_{\hat{\varphi}} \hat{u} \in H_{0}^{1}(\hat{S})$. Using [39] (Cor. 4.1), namely

$$
\left\|\hat{\theta}^{-1} \hat{v}\right\|_{0, \hat{S}} \lesssim|\hat{v}|_{1, \hat{S}} \quad \forall \hat{v} \in H_{0}^{1}(\hat{S})
$$

we conclude

$$
\begin{aligned}
\left|\left[\sin ^{-1} \theta \partial_{\varphi} u\right]\right|_{0, S} & \left.\sim \| \hat{\theta}^{-1} \partial_{\hat{\varphi} \hat{u}}\right]\left.\right|_{0, \hat{S}} \\
& \lesssim\left\|\hat{\theta}^{-1} \partial_{\hat{\varphi}} \hat{u}\right\|_{0, \hat{S}} \\
& \lesssim\left\|\partial_{\hat{\varphi} \hat{\theta}} \hat{u}\right\|_{0, \hat{S}}+\left\|\partial_{\hat{\varphi} \hat{\varphi} \hat{u}}\right\|_{0, \hat{S}} \\
& \lesssim\left\|\hat{\theta}^{-1} \partial_{\hat{\varphi} \hat{\theta}} \hat{u}\right\|_{0, \hat{S}}+\left\|\hat{\theta}^{-2} \partial_{\hat{\varphi} \hat{\varphi} \hat{u}}\right\|_{0, \hat{S}} \\
& \lesssim h_{\theta, S}\left(\left|\left[\sin ^{-1} \theta \partial_{\varphi \theta} u\right]\right|_{0, S}+\left|\left[\sin ^{-2} \theta \partial_{\varphi \varphi} u\right]\right|_{0, S}\right)
\end{aligned}
$$

The desired estimate is obtained by combining (39-41).

Until now we assumed that $[u]_{2, T}<\infty$. In the case of polyhedral corners, however, the eigensolutions do in general not possess this regularity near the corners $\tilde{P}_{e}$ of $\tilde{\Omega}$. Let $\tilde{P}_{e}$ be represented by $P_{e}=\left(\varphi_{0}, \theta_{0}\right)$ and consider first the case $\theta_{0} \notin\{0, \pi\}$. Then we can modify the proof of Lemma 4.8 by using the following result of Raugel [43], see also [15] (Sect. 8.4.1),

$$
\left|u-\mathrm{I}_{h} u\right|_{m, T} \lesssim h_{T}^{2-m-\beta} \sum_{i+j=2}\left\|R_{e}^{\beta} \partial_{\varphi}^{i} \partial_{\theta}^{j} u\right\|_{0, T}, \quad m=0,1
$$

$R_{e}:=R_{e}(\varphi, \theta)=\left[\left(\varphi-\varphi_{0}\right)^{2}+\left(\theta-\theta_{0}\right)^{2}\right]^{1 / 2}, \beta \in[0,1)$. This leads immediately to the following lemma.

Lemma 4.11. Let $T$ be an isotropic triangle and assume $\vartheta_{-, T} \gtrsim 1$. Then the error estimate

$$
\left[u-\mathrm{I}_{h} u\right]_{m, T} \lesssim h_{T}^{2-m-\beta} \sum_{i+j=2}\left|\left[R_{e}^{\beta} \partial_{\varphi}^{i} \partial_{\theta}^{j} u\right]\right|_{0, T}, \quad m=0,1
$$

holds for $\beta \in[0,1)$.

Proof. The error estimate follows from (42) and $\sin \theta \sim 1$ in $T$.

In the case that $\theta_{0}=0$ we have to modify Lemma 4.10 .

Lemma 4.12. Assume that \|\|$u \|_{2, \beta, S}<\infty$ and $1-\operatorname{Re} \alpha_{e}<\beta_{e}<\frac{1}{2}$. Then the interpolation operator $\mathrm{I}_{h}$ is well defined in $S, S$ from (35), and the estimates

$$
\left[u-\mathrm{I}_{h} u\right]_{m, S} \lesssim h_{\theta, S}^{2-m-\beta}\left[\theta^{\beta} u\right]_{2, S}, \quad m=0,1
$$

hold.

Proof. We follow the lines of the proof of Lemma 4.10. It is shown in [33] (Lem. 1.1) that $\|u\|_{C(\bar{S})} \lesssim\|u\|_{2,1 / 2+\beta, S}$ if $\beta_{e}<\frac{1}{2}$. Consequently, $\mathrm{I}_{h}$ is well defined for functions from $V_{1 / 2+\beta}^{2}$. Estimate (38) holds also when $\frac{1}{2}$ is replaced by $\frac{1}{2}+\beta, \beta \in[0,1)$. This can be proved in the same way as in [39]; we have to ensure only that the embedding $H_{1 / 2+\beta}^{2}(\hat{S}) \hookrightarrow H_{1 / 2+\beta}^{1}(\hat{S})$ is compact. (The definition of these weighted spaces is analogous to (36).) This is indeed true, see [22] (Lem. 3.5). 


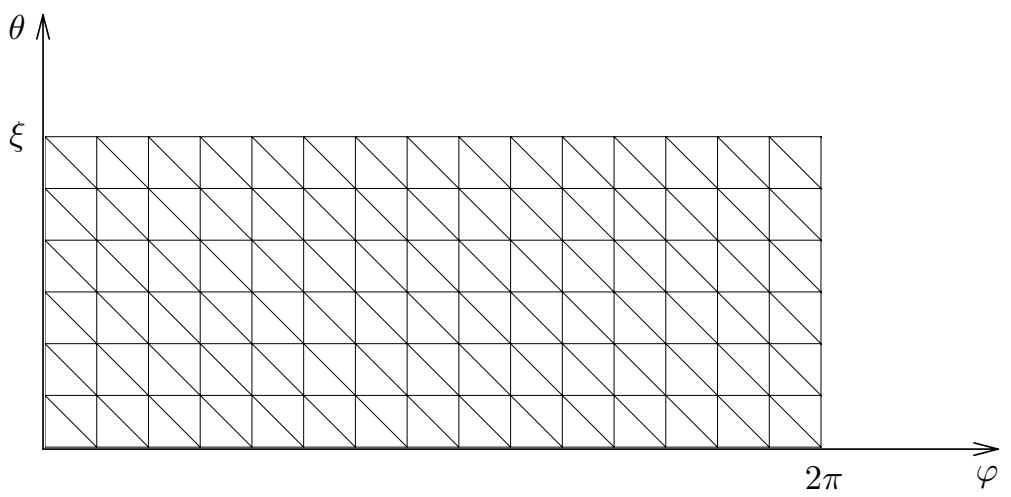

Figure 3. Illustration of the mesh for the conical domain.

For proving the estimate which is analogous to (39) we need the embedding theorem $H_{1 / 2+\beta}^{2}(\hat{S}) \hookrightarrow H_{1 / 2}^{1}(\hat{S})$, which holds for $\beta \in[0,1)$, see also [22] (Lem. 3.5). Consequently,

$$
\begin{aligned}
\left|\left[\partial_{\theta}\left(u-\mathrm{I}_{h} u\right)\right]\right|_{0, S} & \lesssim\left\|\partial_{\hat{\theta}}^{m}\left(\hat{u}-\hat{\mathrm{I}}_{h} \hat{u}\right)\right\|_{0 ; 1 / 2, \hat{S}} \\
& =\inf _{\hat{w} \in \mathcal{P}_{1}}\left\|\partial_{\hat{\theta}}\left(\hat{u}-\hat{w}-\hat{\mathrm{I}}_{h}(\hat{u}-\hat{w})\right)\right\|_{0 ; 1 / 2, \hat{S}} \\
& \lesssim \inf _{\hat{w} \in \mathcal{P}_{1}}\|\hat{u}-\hat{w}\|_{2 ; 1 / 2+\beta, \hat{S}} \\
& \lesssim|\hat{v}|_{2 ; 1 / 2+\beta, \hat{S}} \\
& \lesssim h_{\theta, S}^{1-\beta}\left[\theta^{\beta} u\right]_{2, S} .
\end{aligned}
$$

The derivation of $(40,41)$, is easily modified since all conclusions hold true also when $h_{\theta, T}$ is substituted by $h_{\theta, T}^{1-\beta},\left\|\hat{\theta}^{-1} \partial_{\hat{\varphi} \hat{\theta}} \hat{u}\right\|_{0, \hat{T}}$ by $\left\|\hat{\theta}^{-1+\beta} \partial_{\hat{\varphi} \hat{\theta}} \hat{u}\right\|_{0, \hat{T}},\left\|\hat{\theta}^{-2} \partial_{\hat{\varphi} \hat{\varphi} \hat{u}}\right\|_{0, \hat{T}}$ by $\left\|\hat{\theta}^{-2+\beta} \partial_{\hat{\varphi} \hat{\varphi} \hat{u}}\right\|_{0, \hat{T}}$ etc.

\subsection{Approximation error estimates}

The circular cone is the simplest case since $\Omega$ is a rectangle and the eigenfunctions are regular, see Section 3.2. Let $\left\{\mathcal{T}_{h}\right\}_{h}$ be a sequence of quasi-uniform triangular meshes. Each mesh is obtained from a rectangular mesh by dividing each rectangle into two triangles of diameter $h$, see Figure 3.

Theorem 4.13. Assume that $u \in V$ is a vector function with $[u]_{2, \Omega}<\infty$. Then the interpolation error can be estimated on the mesh described above by

$$
\left\|u-\mathrm{I}_{h} u\right\|_{V} \lesssim h[u]_{2, \Omega}
$$

Proof. We apply the interpolation error estimates of Lemmata 4.8-4.10 and obtain

$$
\left\|u-\mathrm{I}_{h} u\right\|_{V}^{2} \sim \sum_{T \in \mathcal{T}_{h}} \sum_{m=0}^{1}\left[u-\mathrm{I}_{h} u\right]_{m, T}^{2} \lesssim \sum_{m=0}^{1}\left(h^{2}+h\right)[u]_{2, T}^{2} \sim h[u]_{2, \Omega}^{2}
$$

which is the desired result.

In the case of polyhedral vertices, the eigenfunctions have singularities itself, see Section 3.2. On uniform meshes we do not achieve the optimal convergence order, see Remark 4.15. Therefore, we use refined meshes as defined in Section 4.2. 
Theorem 4.14. Assume that $u \in V$ is a vector function with $\|u\|_{2, \beta, \Omega}, \beta \in[0,1)$, where \|\|$^{*} \mid \|_{2, \beta, \Omega}$ was introduced in (22). On the graded meshes introduced in Section 4.2, the interpolation error can be estimated by

$$
\left\|u-\mathrm{I}_{h} u\right\|_{V} \lesssim h\|u\|_{2, \beta, \Omega} .
$$

Proof. If a corner $P_{e}$ is at position $\left(\varphi_{0}, \theta_{0}\right), \theta_{0} \notin\{0, \pi\}$, then the refined mesh in the neighbourhood of $P_{e}$ consists of isotropic triangles only. For all elements $T$ with $\left(\varphi_{0}, \theta_{0}\right) \in \bar{T}$ Lemma 4.11 is applied. By using $2-m-\beta \geq 1-\beta$ and (28) we obtain for $m=0,1$,

$$
\begin{aligned}
{\left[u-\mathrm{I}_{h} u\right]_{m, T} } & \lesssim h_{T}^{1-\beta} \sum_{i+j=2}\left|\left[R_{e}^{\beta} \partial_{\varphi}^{i} \partial_{\theta}^{j} u\right]\right|_{0, T} \\
& \sim h \sum_{i+j=2}\left|\left[R_{e}^{\beta} \partial_{\varphi}^{i} \partial_{\theta}^{j} u\right]\right|_{0, T} \\
& \lesssim h\|u\|_{2, \beta, T} .
\end{aligned}
$$

For all other elements $T$ in the refinement zone, $T \cap N_{e} \neq \emptyset$, we use Lemma 4.8, (28), and dist $\left(T, P_{e}\right)<R_{e}$ for all points of $T$, and obtain for $m=0,1$,

$$
\begin{aligned}
{\left[u-\mathrm{I}_{h} u\right]_{m, T} } & \lesssim h_{T}[u]_{2, T} \\
& \sim h[\operatorname{dist}(P, T)]^{\beta}[u]_{2, T} \\
& \lesssim h\left[R_{e}^{\beta} u\right]_{2, T} \\
& \lesssim h\|u\| \|_{2, \beta, T} .
\end{aligned}
$$

Let now $P_{e}$ be the north pole. Then for all elements $T$ in a strip $S$ as defined between Lemmata 4.9 and 4.10 , Lemma 4.12 is applied. By using (29) and (30) we obtain, again for $m=0,1$,

$$
\begin{aligned}
{\left[u-\mathrm{I}_{h} u\right]_{m, S} } & \lesssim h_{\theta, S}^{1-\beta}\left[\theta^{\beta} u\right]_{2, S} \\
& \sim h\left[\theta^{\beta} u\right]_{2, S} \\
& \lesssim h\|u\|_{2, \beta, S} .
\end{aligned}
$$

For all other elements $T$ with $T \cap N_{e} \neq \emptyset$ we can use Lemma 4.9, $(29,30), \vartheta_{-, T} \lesssim 1$, and $\vartheta_{-, T} \leq \theta$ for all points of $T$, and obtain for $m=0,1$,

$$
\begin{aligned}
{\left[u-\mathrm{I}_{h} u\right]_{m, T} } & \lesssim h_{\varphi, T} \vartheta_{-, T}\left|\left[\sin ^{-2} \theta \partial_{\varphi}^{2} u\right]\right|_{0, T}+\left(h_{\varphi, T} \vartheta_{-, T}+h_{\theta, T}\right)\left|\left[\sin ^{-1} \theta \partial_{\varphi} \partial_{\theta} u\right]\right|_{0, T}+h_{\theta, T}\left|\left[\partial_{\theta}^{2} u\right]\right|_{0, T} \\
& \lesssim h \vartheta_{-, T}^{\beta}[u]_{2, T} \\
& \lesssim h\left[\theta^{\beta} u\right]_{2, T} \\
& \lesssim h\|u\| \|_{2, \beta, T} .
\end{aligned}
$$

If the south pole is also a singular corner of $\tilde{\Omega}$, then it is treated analogously. For elements $T$ outside a refinement region $N_{e}$ we use Lemma 4.8, 4.9 or 4.10, respectively, and obtain also

$$
\left[u-\mathrm{I}_{h} u\right]_{m, T} \lesssim h\|u\|_{2, \beta, T} .
$$

Combining all these estimates we obtain the desired result.

Remark 4.15. If the mesh is not refined, $h_{T}=h$ in (28) and $h_{\theta, T}=h$ in (30), then one can proceed analogously. However, we obtain in (43-46) only a local convergence order $h^{1-\beta}$ (using $h \leq h^{1-\beta}[\operatorname{dist}(P, T)]^{\beta}$ in $(44)$ 
and (46)). That means that the global convergence order is

$$
1-\max _{e} \beta_{e}=\min _{e} \operatorname{Re} \alpha_{e}-\varepsilon, \quad \varepsilon>0 \text { arbitrary }
$$

since $\beta_{e}>1-\operatorname{Re} \alpha_{e}$ was assumed.

Corollary 4.16. For the discretization as described in Section 4.2 the following error estimates for the eigenpairs $\left(\lambda_{0}, u\left(\lambda_{0} ; \cdot\right)\right)$ of $(14)$ hold:

$$
\begin{aligned}
\left|\lambda_{h}-\lambda_{0}\right| & \lesssim h^{2 / \kappa}\left|\lambda_{0}\right|^{4 / \kappa} \\
\left|\hat{\lambda}_{h}-\lambda_{0}\right| & \lesssim h^{2}\left|\lambda_{0}\right|^{4}, \\
\left\|u_{h}-u\left(\lambda_{0} ; \cdot\right)\right\|_{V} & \lesssim h^{\alpha}\left|\lambda_{0}\right|^{2 \alpha}, \quad \alpha=\min \{1,2 / \kappa\} .
\end{aligned}
$$

Proof. Using Theorems 4.13 and 4.14 and defining

$$
\sigma\left(\lambda_{0}\right):=\max _{v \in W\left(\mathcal{B}, \lambda_{0}\right),\|v\|_{V}=1}\left|\|v \mid\|_{2, \beta, \Omega}, \quad \sigma^{*}\left(\lambda_{0}\right):=\max _{v \in W\left(\mathcal{B}^{*}, \lambda_{0}\right),\|v\|_{V}=1}\|v\|_{2, \beta, \Omega},\right.
$$

we find that

$$
\begin{aligned}
& \varepsilon_{h}:=\max _{v \in W\left(\mathcal{B}, \lambda_{0}\right),\|v\|_{V}=1} \varepsilon_{h}(v) \lesssim h \sigma\left(\lambda_{0}\right), \\
& \varepsilon_{h}^{*}:=\max _{v \in W\left(\mathcal{B}^{*}, \lambda_{0}\right),\|v\|_{V}=1} \varepsilon_{h}(v) \lesssim h \sigma^{*}\left(\lambda_{0}\right) .
\end{aligned}
$$

There exist generalized eigenelements $w \in W\left(\mathcal{B}, \lambda_{0}\right)$ and $w^{*} \in W\left(\mathcal{B}^{*}, \lambda_{0}\right),\|w\|_{V}=\left\|w^{*}\right\|_{V}=1$, such that

$$
\sigma\left(\lambda_{0}\right)=\|w\|_{2, \beta, \Omega}, \quad \sigma^{*}\left(\lambda_{0}\right)=\left\|w^{*}\right\|_{2, \beta, \Omega} .
$$

Therefore we can conclude with Lemma 3.12 that

$$
\sigma\left(\lambda_{0}\right) \lesssim\left|\lambda_{0}\right|^{2}, \quad \sigma^{*}\left(\lambda_{0}\right) \lesssim\left|\lambda_{0}\right|^{2}
$$

and, consequently,

$$
\varepsilon_{h} \lesssim h\left|\lambda_{0}\right|^{2}, \quad \varepsilon_{h}^{*} \lesssim h\left|\lambda_{0}\right|^{2}
$$

Using Theorems 4.5-4.7, we derive the desired estimates.

Remark 4.17. The number $\lambda_{0}$ does not depend on $h$ and can thus be considered as a constant factor. But a large eigenvalue $\lambda_{0}$ diminishes the accuracy of its approximation.

\section{Numerical tests}

In order to confirm the theoretically predicted convergence order we test our method in a case where the eigenvalues can be obtained by a simpler method to arbitrary precision, namely we consider the circular cone

$$
K=\left\{(\cos \varphi \sin \theta, \sin \varphi \sin \theta, \cos \theta) \in \mathbb{R}^{3}: 0 \leq \varphi<2 \pi, 0<\theta<\xi\right\}
$$

and isotropic material. The values of $\lambda_{\min }=\lambda_{\min }(\xi)$ are calculated for the Poisson ratio $\nu=0.3$ in $[5,6]$ for rotationally symmetric forces and in [45] for the general case. The eigenvalues satisfy a rather lengthy transcendental equation. In the case $\xi=90^{\circ}$ the cone degenerates to the half-space and the eigenvalues are the positive integers. 
TABLE 1. Example 1: Approximate eigenvalues $\alpha$, error and convergence order for various mesh sizes and angles.

\begin{tabular}{|c|c|c|c|c|}
\hline Angle & $(\pi h)^{-1}$ & $\lambda_{\min }^{h}$ & error & approx. order \\
\hline \multirow[t]{6}{*}{90} & 8 & 0.9917948929 & $8.20511 \mathrm{e}-03$ & \multirow{6}{*}{$\begin{array}{l}1.94 \\
2.02 \\
2.02 \\
2.01\end{array}$} \\
\hline & 16 & 0.9978589231 & $2.14108 \mathrm{e}-03$ & \\
\hline & 32 & 0.9994719803 & $5.28020 \mathrm{e}-04$ & \\
\hline & 64 & 0.9998693746 & $1.30625 \mathrm{e}-04$ & \\
\hline & 128 & 0.9999674776 & $3.25224 \mathrm{e}-05$ & \\
\hline & exact value & 1.00000000 & & \\
\hline \multirow[t]{6}{*}{120} & 8 & $0.5425845623 \pm 0.0054051649 \mathrm{i}$ & $5.44107 \mathrm{e}-03$ & \multirow{6}{*}{$\begin{array}{l}1.88 \\
1.96 \\
1.80 \\
1.99\end{array}$} \\
\hline & 16 & $0.5419631338 \pm 0.0014806910 \mathrm{i}$ & $1.48069 \mathrm{e}-03$ & \\
\hline & 32 & $0.5419310513 \pm 0.0003796528 \mathrm{i}$ & $3.80792 \mathrm{e}-04$ & \\
\hline & 64 & $0.5419564998 \pm 0.0001091573 \mathrm{i}$ & $1.09230 \mathrm{e}-04$ & \\
\hline & 128 & $0.5419571416 \pm 0.0000273475 \mathrm{i}$ & $2.75516 \mathrm{e}-05$ & \\
\hline & exact value & 0.5419604892 & & \\
\hline \multirow[t]{6}{*}{150} & 8 & $0.3037192732 \pm 0.0049080364 \mathrm{i}$ & $4.93810 \mathrm{e}-03$ & \multirow{6}{*}{$\begin{array}{l}1.64 \\
1.96 \\
1.94 \\
2.02\end{array}$} \\
\hline & 16 & $0.3036384809 \pm 0.0015122140 \mathrm{i}$ & $1.58161 \mathrm{e}-03$ & \\
\hline & 32 & $0.3032978815 \pm 0.0003872941 \mathrm{i}$ & $4.06278 \mathrm{e}-04$ & \\
\hline & 64 & $0.3032079364 \pm 0.0001010282 \mathrm{i}$ & $1.06218 \mathrm{e}-04$ & \\
\hline & 128 & $0.3031821396 \pm 0.0000252988 \mathrm{i}$ & $2.62490 \mathrm{e}-05$ & \\
\hline & exact value & 0.30317514090 & & \\
\hline
\end{tabular}

In the test we used a fifth order numerical integration rule with 7 points to generate the matrices. The eigenvalue problem is solved with SHIRA, the Skew-Hamiltonian Implicitly Restarted Arnoldi method [3,38], with the shift value $\tau=0.3$ and the convergence criterion $\epsilon=10^{-12}$. The software was mainly written by Uwe Reichel and Cornelia Pester with the help of David S. Watkins. It makes use of various packages like ARPACK [29], Super-LU [12], LAPACK [1], as well as libraries of the Chemnitz SPC group for assembling matrices, graphics, memory management and other basic tasks [16, 42].

In Table 1 we display the smallest approximate eigenvalue and the error for various mesh sizes and angles $\xi \in\left\{90^{\circ}, 120^{\circ}, 150^{\circ}\right\}$. From the results of two successive levels of refinement we compute the approximate convergence order.

We see that the relative error is nearly independent of the angle and the approximation order is surprisingly close to the predicted order two. The case $\xi=120^{\circ}$ is also calculated in [14] by using an adaptive finite element method and by exploiting the symmetry in the eigensolutions. There, the approximate convergence order 2.12 was obtained for linear finite elements and 4.60 for quadratic elements.

As a second test we consider the Fichera corner. The domain can be described as the unit sphere where one octant is missing. A representation in the parameter plane is shown in Figure 4. This problem has been considered in the literature for isotropic material, for example in [46] for a single material and Dirichlet boundary conditions and in [13] for a bi-material joint and Neumann boundary conditions. We use also the bi-material 

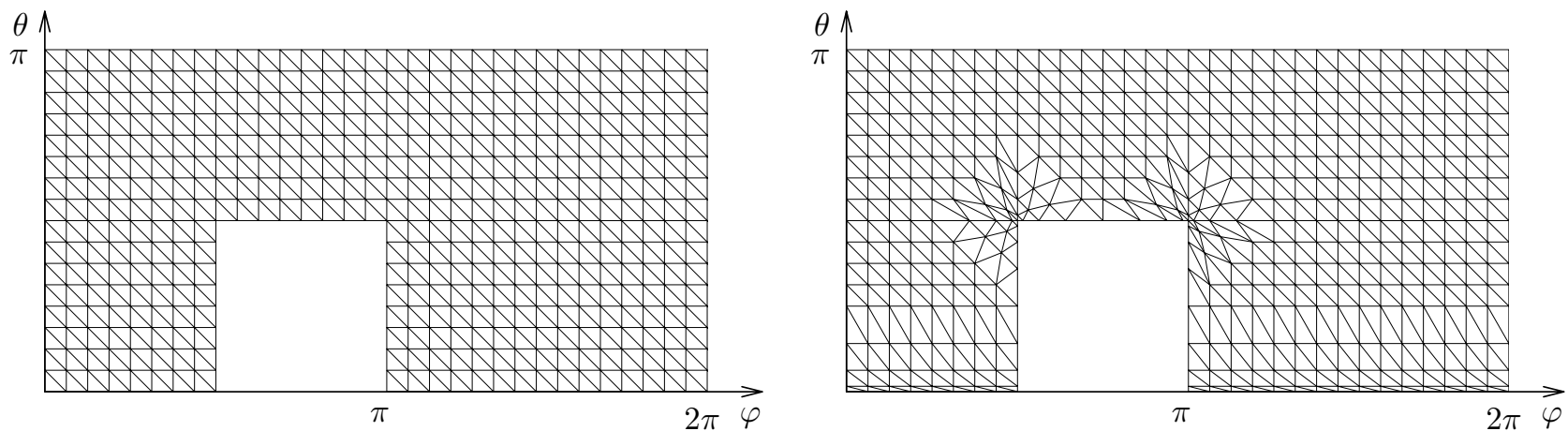

FigURE 4. Fichera corner in the parameter plane: uniform mesh (left) and graded mesh (right).

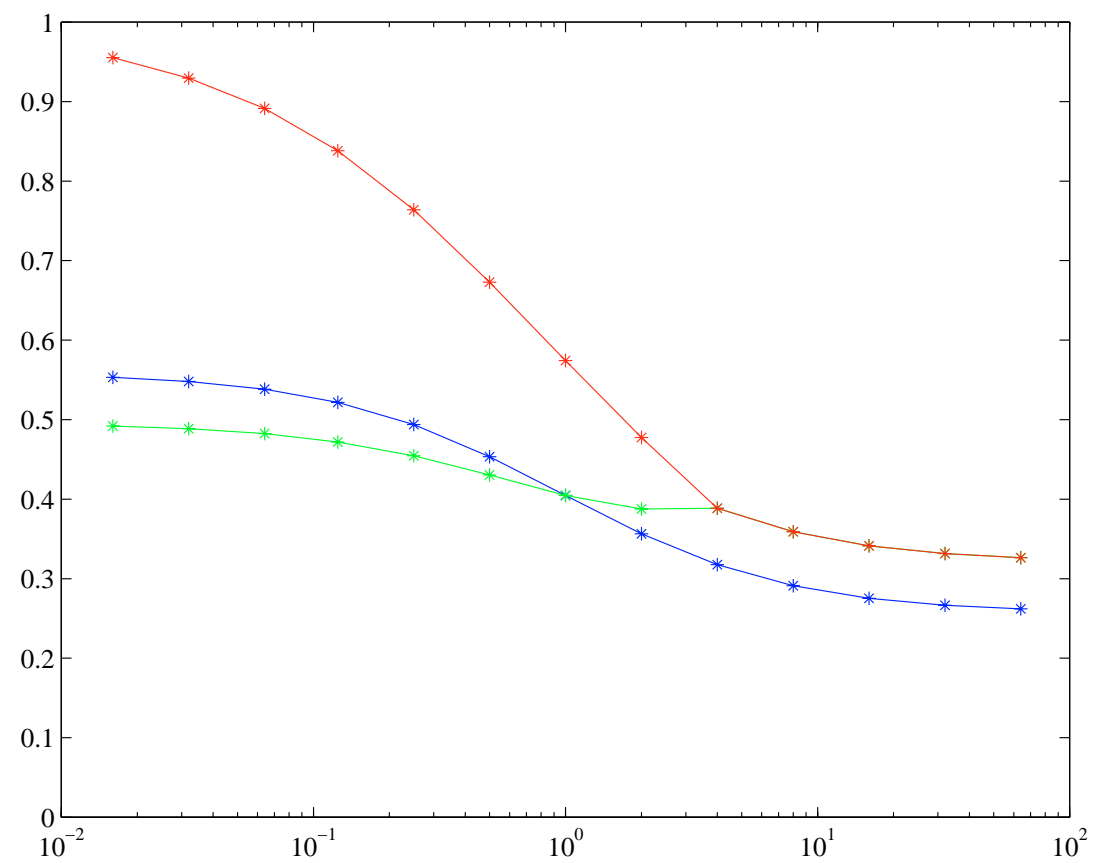

FiguRE 5. Three minimal eigenvalues $\alpha$ for various material parameters.

joint but calculate the eigenvalues for the Dirichlet problem. The material is defined by

$$
\nu=0.3, \quad E= \begin{cases}E_{1}=1.0 & \text { for } \theta<\frac{1}{2} \pi, \\ E_{2} & \text { for } \theta>\frac{1}{2} \pi,\end{cases}
$$

where $E_{2}$ is varied in the interval $[0.016,64]$. The three smallest eigenvalues are plotted against $E_{2}$ in Figure 5 .

The exact eigenvalues are not known in this case. Therefore we estimate the convergence order by calculating with three different meshes of mesh size $h, \frac{1}{2} h$, and $\frac{1}{4} h$. By assuming $\lambda_{h}-\lambda=C, \lambda_{h / 2}-\lambda=C 2^{-z}$ and $\lambda_{h / 4}-\lambda=C 2^{-2 z}$ we obtain the estimated convergence order $z=\log _{2}\left[\left(\lambda_{h}-\lambda_{h / 2}\right) /\left(\lambda_{h / 2}-\lambda_{h / 4}\right)\right]$. Table 2 shows the results for uniform meshes and meshes graded with the parameter $\beta=0.5$. This choice of $\beta$ should 
TABLE 2. Approximate minimum eigenvalues $\alpha$ for different mesh sizes and various material parameters, for both uniform (above) and graded meshes (below).

\begin{tabular}{cccccccc}
\hline$E_{2}$ & 0.016 & 0.0625 & 0.025 & 1.0 & 4.0 & 16.0 & 64.0 \\
\hline$h=\pi / 16$ & 0.5017 & 0.4920 & 0.4633 & 0.4123 & 0.3282 & 0.2880 & 0.2755 \\
$h=\pi / 32$ & 0.4957 & 0.4862 & 0.4581 & 0.4078 & 0.3223 & 0.2813 & 0.2685 \\
$h=\pi / 64$ & 0.4933 & 0.4839 & 0.4560 & 0.4059 & 0.3196 & 0.2781 & 0.2650 \\
$h=\pi / 128$ & 0.4924 & 0.4829 & 0.4551 & 0.4051 & 0.3184 & 0.2765 & 0.2633 \\
\hline est. order & 1.3354 & 1.3274 & 1.2951 & 1.2396 & 1.1418 & 1.0420 & 1.0062 \\
\hline$h=\pi / 16$ & 0.4974 & 0.4878 & 0.4598 & 0.4096 & 0.3262 & 0.2857 & 0.2729 \\
$h=\pi / 32$ & 0.4933 & 0.4838 & 0.4560 & 0.4060 & 0.3203 & 0.2788 & 0.2656 \\
$h=\pi / 64$ & 0.4922 & 0.4827 & 0.4549 & 0.4049 & 0.3183 & 0.2762 & 0.2629 \\
$h=\pi / 128$ & 0.4919 & 0.4824 & 0.4546 & 0.4046 & 0.3177 & 0.2754 & 0.2620 \\
\hline est. order & 1.9343 & 1.9349 & 1.9062 & 1.8556 & 1.7092 & 1.6233 & 1.5828 \\
\hline
\end{tabular}

be sufficient for theoretical purposes since the edge singular exponent is always larger then 0.5 . As a numerical computation has shown this limit is reached here for $E_{2} \rightarrow \infty$.

We see that graded meshes deliver a more accurate eigenvalue approximation when the same number of unknowns is used. We see also that the estimated convergence order is significantly larger for graded meshes. The optimal convergence order is not yet reached. However, we are not able to do calculations with a still smaller mesh size.

\section{REFERENCES}

[1] E. Anderson, Z. Bai, C. Bischof, S. Blackford, J. Demmel, J. Dongarra, J. Du Croz, A. Greenbaum, S. Hammarling, A. McKenney and D. Sorensen, LAPACK Users' Guide. SIAM, Philadelphia, PA, third edition (1999).

[2] T. Apel, Anisotropic finite elements: Local estimates and applications. Teubner, Stuttgart, Adv. Numer. Math. (1999). Habilitationsschrift.

[3] T. Apel, V. Mehrmann and D. Watkins, Structured eigenvalue methods for the computation of corner singularities in 3D anisotropic elastic structures. Comput. Methods Appl. Mech. Engrg. (to appear), Preprint SFB393/01-25, TU Chemnitz (2001).

[4] R.E. Barnhill and J.A. Gregory, Interpolation remainder theory from Taylor expansions on triangles. Numer. Math. 25 (1976) 401-408.

[5] Z.P. Bažant and L.M. Keer, Singularities of elastic stresses and of harmonic functions at conical notches or inclusions. Internat. J. Solids Structures 10 (1974) 957-964.

[6] A.E. Beagles and A.-M. Sändig, Singularities of rotationally symmetric solutions of boundary value problems for the Lamé equations. ZAMM 71 (1990) 423-431.

[7] P. Benner, R. Byers, V. Mehrmann and H. Xu, Numerical computation of deflating subspaces of embedded Hamiltonian pencils. SIAM J. Matrix Anal. Appl. (to appear), Preprint SFB393/99-15, TU Chemnitz (1999).

[8] M. Costabel and M. Dauge, General edge asymptotics of solutions of second order elliptic boundary value problems I, II. Proc. Roy. Soc. Edinburgh Sect. A 123 (1993) 109-155, 157-184.

[9] M. Dauge, Elliptic boundary value problems on corner domains - smoothness and asymptotics of solutions. Lecture Notes in Math. 1341, Springer, Berlin (1988).

[10] M. Dauge, Singularities of corner problems and problems of corner singularities, in: Actes du 30ème Congrés d'Analyse Numérique: CANum '98 (Arles, 1998), Soc. Math. Appl. Indust., Paris (1999) 19-40.

[11] M. Dauge, "Simple" corner-edge asymptotics. Internet publication, http://www.maths.univ-rennes1.fr/ dauge/publis/corneredge.pdf (2000).

[12] J.W. Demmel, J.R. Gilbert and X.S. Li, SuperLU Users' Guide. Technical Report LBNL-44289, Lawrence Berkeley National Laboratory (1999).

[13] A. Dimitrov, H. Andrä and E. Schnack, Efficient computation of order and mode of corner singularities in 3d-elasticity. Internat. J. Numer. Methods Engrg. 52 (2001) 805-827. 
[14] A. Dimitrov and E. Schnack, Asymptotical expansion in non-Lipschitzian domains: a numerical approach using $h$-fem. Numer. Linear Algebra Appl. (to appear).

[15] P. Grisvard, Elliptic problems in nonsmooth domains. Pitman, Boston-London-Melbourne, Monographs and Studies in Mathematics 21 (1985).

[16] G. Haase, T. Hommel, A. Meyer, and M. Pester, Bibliotheken zur Entwicklung paralleler Algorithmen. Preprint SPC95_20, TU Chemnitz-Zwickau (1995). Updated version of SPC94_4 and SPC93_1.

[17] H. Jeggle and E. Wendland, On the discrete approximation of eigenvalue problems with holomorphic parameter dependence. Proc. Roy. Soc. Edinburgh Sect. A 78 (1977) 1-29.

[18] O.O. Karma, Approximation of operator functions and convergence of approximate eigenvalues. Tr. Vychisl. Tsentra Tartu. Gosudarst. Univ. 24 (1971) 3-143. In Russian.

[19] O.O. Karma, Asymptotic error estimates for approximate characteristic value of holomorphic Fredholm operator functions. Zh. Vychisl. Mat. Mat. Fiz. 11 (1971) 559-568. In Russian.

[20] O.O. Karma, Approximation in eigenvalue problems for holomorphic Fredholm operator functions. I. Numer. Funct. Anal. Optim. 17 (1996) 365-387.

[21] O.O. Karma, Approximation in eigenvalue problems for holomorphic Fredholm operator functions. II: Convergence rate. Numer. Funct. Anal. Optim. 17 (1996) 389-408.

[22] V.A. Kondrat'ev, Boundary value problems for elliptic equations on domains with conical or angular points. Tr. Mosk. Mat. Obs. 16 (1967) 209-292. In Russian.

[23] V.A. Kozlov, V.G. Maz'ya and J. Roßmann, Elliptic Boundary Value Problems in Domains with Point Singularities. American Mathematical Society (1997).

[24] V.A. Kozlov, V.G. Maz'ya and J. Roßmann, Spectral Problems Associated with Corner Singularities of Solutions to Elliptic Equations. American Mathematical Society (2001).

[25] S.G. Krejn and V.P. Trofimov, On holomorphic operator functions of several complex variables. Funct. Anal. Appl. 3 (1969) 85-86. In Russian. English transl. in Funct. Anal. Appl. 3 (1969) 330-331.

[26] S.G. Krejn and V.P. Trofimov, On Fredholm operator depending holomorphically on the parameters. Tr. Seminara po funk. anal. Voronezh univ. (1970) 63-85.

[27] D. Leguillon, Computation of 3D-singularities in elasticity, in: Boundary value problems and integral equations in nonsmooth domains, M. Costabel, M. Dauge and S. Nicaise Eds. New York, Lecture Notes in Pure and Appl. Math. 167 (1995) 161-170. Marcel Dekker. Proceedings of a conference at CIRM, Luminy, France, May 3-7 (1993).

[28] D. Leguillon and E. Sanchez-Palencia, Computation of singular solutions in elliptic problems and elasticity. Masson, Paris (1987).

[29] R.B. Lehoucq, D.C. Sorensen and C. Yang, ARPACK user's guide. Solution of large-scale eigenvalue problems with implicitly restarted Arnoldi methods. SIAM, Philadelphia, PA, Software Environ. Tools 6 (1998).

[30] A.S. Markus, On holomorphic operator functions. Dokl. Akad. Nauk 119 (1958) 1099-1102. In Russian.

[31] A.S. Markus, Introduction to spectral theory of polynomial operator pencils. American Mathematical Society, Providence (1988).

[32] A.S. Markus and E.I. Sigal, The multiplicity of the characteristic number of an analytic operator function. Mat. Issled. 5 (1970) 129-147. In Russian.

[33] V.G. Maz'ya and B. Plamenevskiǔ, $L_{p}$-estimates of solutions of elliptic boundary value problems in domains with edges. $T r$. Mosk. Mat. Obs. 37 (1978) 49-93. In Russian. English transl. in Trans. Moscow Math. Soc. 1 (1980) 49-97.

[34] V.G. Maz'ya and B. Plamenevskiŭ, The first boundary value problem for classical equations of mathematical physics in domains with piecewise smooth boundaries, part I, II. Z. Anal. Anwendungen 2 (1983) 335-359, 523-551. In Russian.

[35] V.G. Maz'ya and J. Roßmann, Über die Asymptotik der Lösung elliptischer Randwertaufgaben in der Umgebung von Kanten. Math. Nachr. 138 (1988) 27-53.

[36] V.G. Maz'ya and J. Roßmann, On the Agmon-Miranda maximum principle for solutions of elliptic equations in polyhedral and polygonal domains. Ann. Global Anal. Geom. 9 (1991) 253-303.

[37] V.G. Maz'ya and J. Roßmann, On the behaviour of solutions to the dirichlet problem for second order elliptic equations near edges and polyhedral vertices with critical angles. Z. Anal. Anwendungen 13 (1994) 19-47.

[38] V. Mehrmann and D. Watkins, Structure-preserving methods for computing eigenpairs of large sparse skew-Hamiltonian/ Hamiltonian pencils. SIAM J. Sci. Comput. 22 (2001) 1905-1925.

[39] B. Mercier and G. Raugel, Résolution d'un problème aux limites dans un ouvert axisymétrique par éléments finis en $r, z$ et séries de Fourier en $\theta$. RAIRO Anal. Numér. 16 (1982) 405-461.

[40] S.A. Nazarov and B.A. Plamenevsky, Elliptic problems in domains with piecewise smooth boundary. Walter de Gruyter, Berlin, Exposition. Math. 13 (1994).

[41] S. Nicaise, Regularity of the solutions of elliptic systems in polyhedral domains. Bull. Belg. Math. Soc. Simon Stevin 4 (1997) 411-429.

[42] M. Pester, Grafik-Ausgabe vom Parallelrechner für 2D-Gebiete. Preprint SPC94_24, TU Chemnitz-Zwickau (1994).

[43] G. Raugel, Résolution numérique de problèmes elliptiques dans des domaines avec coins. Ph.D. thesis, Université de Rennes, France (1978). 
[44] G. Raugel, Résolution numérique par une méthode d'éléments finis du problème de Dirichlet pour le Laplacien dans un polygone. C. R. Acad. Sci. Paris Sér. I Math. 286 (1978) A791-A794.

[45] A.-M. Sändig and R. Sändig, Singularities of non-rotationally symmetric solutions of boundary value problems for the Lamé equations in a three dimensional domain with conical points. Breitenbrunn, Analysis on manifolds with singularities (1990), Teubner-Texte zur Mathematik, Band 131 (1992) 181-193.

[46] H. Schmitz, K. Volk and W.L. Wendland, On three-dimensional singularities of elastic fields near vertices. Numer. Methods Partial Differential Equations 9 (1993) 323-337.

[47] V. Staroverov, G. Kobelkov, E. Schnack and A. Dimitrov, On numerical methods for flat crack propagation. IMF-Preprint 99-2, Universität Karlsruhe (1999).

[48] V.P. Trofimov, The root subspaces of operators that depend analytically on a parameter. Mat. Issled. 3 (1968) 117-125. In Russian.

[49] G.M. Vainikko and O.O. Karma, Convergence rate of approximate methods in an eigenvalue problem with a parameter entering nonlinearly. Zh. Vychisl. Mat. Mat. Fiz. 14 (1974) 1393-1408. In Russian.

To access this journal online:

www.edpsciences.org 